\title{
Oil maturity assessment using maturity indicators based on methylated dibenzothiophenes
}

\author{
Li Meijun ${ }^{1,2 *}$, Wang T.-G ${ }^{1,2}$, Shi Shengbao, ${ }^{1,2}$, Zhu Lei $^{1,2}$ and Fang Ronghui ${ }^{1,2}$ \\ ${ }^{1}$ State Key Laboratory of Petroleum Resources and Prospecting, China University of Petroleum, Beijing 102249, China \\ ${ }^{2}$ College of Geosciences, China University of Petroleum, Beijing 102249, China \\ (C) China University of Petroleum (Beijing) and Springer-Verlag Berlin Heidelberg 2014
}

\begin{abstract}
Aromatic fractions of 140 oils and condensates that originated from different types of source rocks (marine shale, terrestrial shale and marine carbonate) were analyzed using gas chromatographymass spectrometry (GC-MS) to investigate the relative distributions of methylated dibenzothiophenes with respect to thermal maturity. The positions of methyl groups of trimethyldibenzothiophene isomers (TMDBTs) including those used in the definition of maturity indicator TMDBT index in previous studies were firmly identified by co-elution of internal standards in GC-MS analysis and by comparing with reported retention indices. A new maturity ratio related to dimethyldibenzothiophenes (DMDBTs) is proposed on the basis of the differences in thermodynamic stability among different DMDBT isomers. Another maturity index (TMDBT-I2) based on TMDBTs is also suggested on the basis of our empirical observations and presumed thermodynamic stability of TMDBT isomers. These two newly proposed $(2,6+3,6)-/ 1,4$-DMDBT ratio and TMDBT-I2 correlate well with MDR (4-/1-methyldibenzothiophene) and 2,4-/1,4-DMDBT ratios, suggesting their common chemical reaction mechanisms and similar behavior with increasing maturity. Therefore, they can be effectively applied for maturity assessments. Furthermore, the TMDBTs related maturity parameters are more reliable for over-mature oils and condensates due to the relatively higher concentrations of thermodynamically unstable TMDBT isomers, i.e. 1,4,6-, 1,4,8- and 3,4,6-TMDBT in this study than those of 1-methyldibenzothiophene (1-MDBT) or 1,4-DMDBT. In contrast with 4,6-/1,4-DMDBT, the newly proposed $(2,6+3,6)-/ 1,4$-DMDBT ratios for oils that originated from different types of source rocks have approximately same relationship with the oil maturity $\left(R_{\mathrm{c}} \%\right)$. This suggests that the lithology and organic facies may have relatively less influence on $(2,6+3,6)-/ 1,4$-DMDBT ratio compared to 4,6-/1,4-DMDBT. The maturity parameters based on methylated dibenzothiophenes are particularly useful in the maturity assessments of post- and over-mature oils and condensates and can complement maturity indicators based on steranes and terpanes.
\end{abstract}

Key words: Dibenzothiophene, maturity indicator, molecular thermodynamic stability, sedimentary organic matter

\section{Introduction}

Polycyclic aromatic sulfur heterocyclics (PASHs), such as benzothiophenes (BTs) and dibenzothiophenes (DBTs) are important organosulfur compounds in oils and ancient organic matter. In spite of the debate on the specific source precursors for PASHs and poor knowledge of the chemical mechanisms and kinetics of their formation during diagenesis, distributions of these compounds have been widely used as organic facies and maturity indicators of petroleum and source rocks (e.g. Ho et al, 1974; Radke et al, 1986; Radke, 1988; Budzinski et al, 1991; Hughes et al, 1995; Chakhmakhchev et al, 1997;

*Corresponding author. email: meijunli2008@hotmail.com

Received February 21, 2013
Santamaría-Orozco et al, 1998; Shen et al, 1998; Asif et al, 2009). Recently, the absolute concentrations of total DBTs and the relative abundances of methylated DBT isomers in petroleums are also suggested to be potential geochemical indicators for tracing petroleum migration pathways (Wang et al, 2004; Li et al, 2008a).

Compounds with thiophenic ring structures in kerogen are probably the precursors of BT, DBT and their homologues in oils (Orr, 1986). Proteins are among the sulfur-bearing constituents of organisms from which the kerogen was formed. Due to the insufficient amount of sulfur in plants and animals to produce the large amounts of sulfur compounds in petroleum and sedimentary organic matter, extra sulfur must have been incorporated into the organic matter from source rocks, especially from high sulfur source rocks (Gransch and 
Posthuma, 1974 and references therein). A thermal reaction between elemental sulfur and the organic matter of sediments was proposed to be responsible for the genesis of the sulfurcontaining organic compounds (Hanson, 1960; Douglas and Mair, 1965). Recently, laboratory simulation experiments showed that biphenyls and sulfur can form dibenzothiophenes with carbon catalysis (Xia and Zhang, 2002; Asif et al, 2009). This suggests that the widespread distributions of dibenzothiophene and alkylated dibenzothiophenes in sediments and petroleums may be the result of a catalytic reaction of biphenyl ring systems and surface-adsorbed sulfur on the surface of carbonaceous material (Asif et al, 2009; Li et al, 2013a; 2013b). Abundant BTs and DBTs in petroleum and sedimentary organic matter are commonly associated with anoxic and sulfur-rich depositional environments (Hughes, 1984; Hughes et al, 1995; Radke et al, 2000; Li et al, 2011). The cross plots of dibenzothiophenes/phenanthrene (DBT/ Phen) vs. pristane/phytane $(\mathrm{Pr} / \mathrm{Ph})$ ratios (Hughes et al, 1995), alkylated dibenzothiophene/alkylated dibenzofuran (ADBT/ ADBF) ratios (Radke et al, 2000) and the ternary diagram of the relative abundance of fluorenes, dibenzothiophenes and dibenzofurans ( $\mathrm{Li}$ et al, 2013c) have been introduced as indicators of the depositional environment and lithology of petroleum source rocks.

The molecular thermodynamic stability of methylated dibenzothiophenes varies with the positions of the methyl groups (Radke, 1988; Budzinski et al, 1991; Richard, 2001). Thus, the distribution and relative abundances of methylated DBT isomers can be used as maturity indicators for both crude oils and source rocks (Radke et al, 1986; Radke, 1988; Schou and Myhr, 1988; Budzinski et al, 1991; Radke et al, 1991; Radke and Willsch, 1994; Bao et al, 1996; Chakhmakhchev et al, 1997; Santamaría-Orozco et al, 1998; Shen et al, 1998; Kruge, 2000). Some maturity indicators on the basis of methyldibenzothiophene (MDBT), dimethyldibenzothiophene (DMDBT) and ethyldibenzothiophene (EDBT) such as MDR (4-/1-MDBT ratio) (Radke et al, 1986; Radke, 1988), DBTI $((2+3)-\mathrm{MDBT} / 2 \times(1-\mathrm{MDBT}))$ (Payzant et al, 1989), MDR' $(4-/(4+1)-\mathrm{MDBT})$ and EDR' (4,6-DMDBT/(4-EDBT + 4,6-DMDBT)) (Radke and Willsch, 1994), 4,6-/1,4-DMDBT and 2,4-/1,4-DMDBT ratios (Chakhmakhchev et al, 1997; Santamaría-Orozco et al, 1998) were proposed to assess the thermal maturity of petroleum and organic matter. These maturity parameters change in a regular fashion with increasing maturity up to high levels. Therefore, they are effective maturity indicators, and particularly useful for high to over-mature oils and condensates lacking biomarkers (Chakhmakhchev et al, 1997). Some discrepancies in calibration of the MDR maturity parameter with vitrinite reflectance $\left(R_{\mathrm{o}}\right)$ have also been reported (Dzou et al, 1995). Chakhmakhchev et al (1997) attributed these discrepancies to differences in analytical procedures and/or organic facies control in different geological settings. Huang and Pearson (1999) showed that high MDRs are associated with fresh water source deposition whilst low MDRs are found in oils of hypersaline origin in the Bohai Bay Basin, East China.

The empirical TMDBT index based on the relative abundances of some trimethyldibenzothiophene (TMDBT) isomers (peak 3 and peak 5) in $m / z 226$ mass chromatograms (Fig. 1) was introduced by Chakhmakhchev et al (1997). The TMDBT index correlates well with the MDR and other DBTbased maturity indicators, suggesting its similar behavior with increasing maturity. However, the specific positions of these three methyl groups for those TMDBT isomers were not assigned due to the lack of internal standards and reference retention indices at that time.
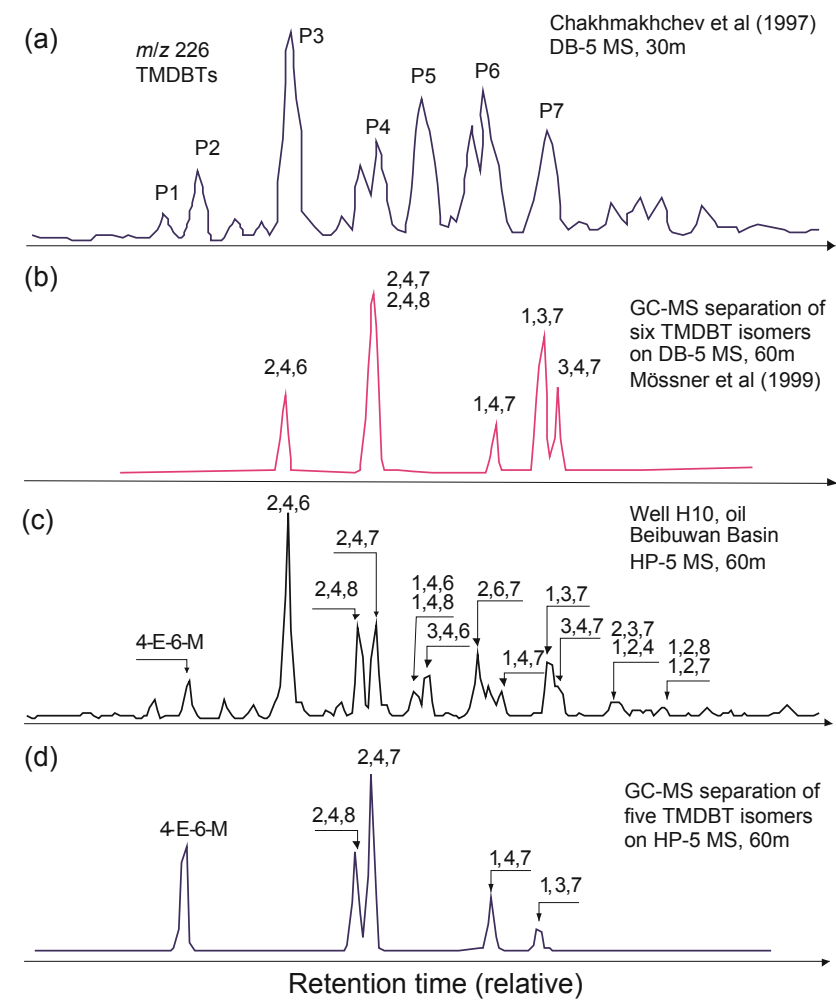

Fig. 1 Identification of trimethyldibenzothiophenes in crude oils. Numbers on peaks indicate the methyl substitution patterns on dibenzothiophene

In this study, we firmly identify a series of TMDBT isomers including peak 3 and peak 5 compounds assigned by Chakhmakhchev et al (1997) by co-elution of internal standards and by comparison of the calculated retention indices (Fig. 1) with those reported in references (Lee et al, 1979; Mössner et al, 1999; Schade and Andersson, 2006). Another TMDBT based maturity parameter is proposed on the basis of our empirical observations and in the light of the differences in thermodynamic stability between different TMDBT isomers. Furthermore, a new DMDBT maturity indicator is also suggested. A large data set, comprising 50 oils and condensates originated from typical terrestrial source rocks, 45 oils from marine shales, and 45 oils from marine carbonates was utilized in this study. The results obtained may provide additional geochemical parameters for maturity evaluation, oil-to-oil and oil-to-source correlations, especially for the characterization of light oils and condensates with low concentrations of biomarkers. The newly proposed DMDBT maturity parameters have some advantages, as they may be more organic facies independent and more reliable for overmature petroleum. 


\section{Samples and methods}

\subsection{Samples}

A total of 50 oil samples collected from the Fushan Depression, Beibuwan Basin (South China Sea) were analyzed for the investigation of DBTs and biomarkers distributions. The Fushan Depression is one of the many Mesozoic-Cenozoic rifting half-grabens in the northern continental shelf of the South China Sea. The discovered oil pools mainly occur in the third $\left(\mathrm{E}_{2} l_{3}\right)$ and first $\left(\mathrm{E}_{2} l_{1}\right)$ members of the Eocene Liushagang Formation ( $\mathrm{Li}$ et al, 2008b). Oils are dominated by light oils or condensates with an average gravity of $47^{\circ}$ (API) (Table 1). They are typical terrestrialsourced high-mature oils with a highest $R_{\mathrm{c}} \%$ equivalent of 1.0-1.1 (Li et al, 2007a; 2007b). The biomarkers are present in very low concentrations or below the detection limit in GCMS analyses ( $\mathrm{Li}$ et al, 2010). The light oils and condensates were derived from type II to III kerogen in source rocks of the second member of the Eocene Liushagang Formation (Ding et al, 2003; Li et al, 2008c).

Table 1 The methylated dibenzothiophenes related maturity parameters in oils from the Beibuwan Basin, South China Sea

\begin{tabular}{|c|c|c|c|c|c|c|c|c|c|c|c|c|c|c|c|c|c|}
\hline $\begin{array}{c}\text { Sample } \\
\text { No. }\end{array}$ & Well & Depth, m & API, ${ }^{\circ}$ & $4-/ 1-$ & $\begin{array}{l}4,6-/ \\
1,4-\end{array}$ & $\begin{array}{c}2,4-1 \\
1,4-\end{array}$ & $\begin{array}{c}(2,6+3,6) / \\
1,4- \\
\end{array}$ & TMDBT-I1 & TMDBT-I2 & MPR & MPI-1 & $\begin{array}{l}R_{\mathrm{c}} \\
\% \\
\end{array}$ & $\begin{array}{l}\mathrm{Ts} / \\
\mathrm{Tm}\end{array}$ & $\begin{array}{c}22 \mathrm{~S} / \\
(22 \mathrm{~S}+22 \mathrm{R})\end{array}$ & $\begin{array}{l}\mathrm{Pr} / \\
\mathrm{Ph}\end{array}$ & $\begin{array}{l}\text { ADBT/ } \\
\text { ADBF }\end{array}$ & $\begin{array}{c}\text { Oleanane } \\
\mathrm{C}_{30} \mathrm{H} \\
\end{array}$ \\
\hline FS1 & H1 & $3151.2-3153.4$ & 45 & 8.31 & 2.07 & 1.77 & 4.23 & 2.36 & 2.11 & 1.48 & 0.90 & 0.94 & 2.04 & 0.60 & 3.29 & 0.76 & 0.41 \\
\hline FS2 & H1-1 & $3347.2-3401.1$ & 46 & 8.03 & 2.07 & 1.51 & 4.07 & 2.59 & 1.99 & 1.43 & 0.86 & 0.92 & 8.70 & 0.65 & 3.69 & 0.81 & 0.56 \\
\hline FS3 & $\mathrm{H} 2$ & $2970.6-3014.0$ & 49 & 7.41 & 1.83 & 1.51 & 3.81 & 2.70 & 2.09 & 1.31 & 0.83 & 0.90 & 3.05 & 0.61 & 3.97 & 0.48 & 0.32 \\
\hline FS4 & $\mathrm{H} 2-1$ & 2909.8-2954.6 & 57 & 6.10 & 1.89 & 1.36 & 3.42 & 2.54 & 1.90 & 1.35 & 0.82 & 0.89 & 5.85 & 0.62 & 3.68 & 0.56 & 0.38 \\
\hline FS5 & $\mathrm{H} 2-2$ & $2973.0-3021.0$ & 46 & 5.67 & 1.76 & 1.38 & 3.24 & 2.64 & 2.06 & 1.26 & 0.76 & 0.86 & 3.31 & 0.60 & 3.71 & 0.51 & 0.31 \\
\hline FS6 & $\mathrm{H} 2-3$ & $2994.6-3084.0$ & 55 & 9.26 & 3.07 & 2.33 & 5.38 & 2.84 & 1.76 & 1.37 & 0.82 & 0.89 & 3.38 & 0.65 & 3.97 & 0.47 & 0.38 \\
\hline FS7 & $\mathrm{H} 3$ & $3015.0-3182.6$ & 51 & 6.69 & 1.59 & 1.32 & 3.48 & 1.92 & 1.86 & 1.18 & 0.76 & 0.86 & 3.04 & 0.52 & 3.49 & 0.69 & 0.37 \\
\hline FS8 & H3-1 & $3949.6-3286.2$ & 43 & 6.93 & 1.83 & 1.55 & 3.61 & 2.42 & 1.85 & 1.31 & 0.78 & 0.87 & 6.40 & 0.62 & 3.76 & 0.62 & 0.44 \\
\hline FS9 & $\mathrm{H} 3-2$ & $3087.0-3144.6$ & 57 & 6.08 & 1.47 & 1.20 & 3.15 & 2.05 & 1.59 & 1.26 & 0.76 & 0.86 & 4.23 & 0.58 & 3.83 & 0.54 & 0.30 \\
\hline FS10 & $\mathrm{H} 3-3$ & $3158.6-3221.0$ & 47 & 5.85 & 1.59 & 1.43 & 3.27 & 2.06 & 1.73 & 1.23 & 0.77 & 0.86 & 4.37 & 0.60 & 3.74 & 0.54 & 0.37 \\
\hline FS11 & HX4 & $3253.0-3259.6$ & 41 & 5.14 & 1.23 & 1.02 & 2.40 & 1.60 & 1.56 & 0.98 & 0.66 & 0.79 & 2.61 & 0.61 & 4.00 & 0.77 & 0.62 \\
\hline FS12 & HX4 & $2965.5-3073.4$ & 43 & 6.64 & 1.73 & 1.43 & 3.32 & 2.55 & 2.00 & 1.26 & 0.74 & 0.84 & 3.83 & 0.59 & 4.00 & 0.49 & 0.28 \\
\hline FS13 & H4-1 & $2851.0-2862.2$ & 53 & 7.55 & 1.53 & 1.34 & 3.16 & 2.77 & 2.14 & 1.17 & 0.75 & 0.85 & 2.77 & 0.60 & 3.99 & 0.44 & 0.26 \\
\hline FS14 & H5 & $3008.6-3011.8$ & 44 & 4.75 & 1.21 & 1.01 & 2.53 & 1.81 & 1.42 & 0.98 & 0.65 & 0.79 & 3.67 & 0.62 & 3.93 & 0.45 & 1.02 \\
\hline FS15 & H5 & $2715.8-2731.0$ & 53 & 9.55 & 2.50 & 1.92 & 4.71 & 3.23 & 2.56 & 1.55 & 0.92 & 0.95 & 3.25 & 0.49 & 3.93 & 0.74 & 0.51 \\
\hline FS16 & H6 & 2884.6-2899.0 & 39 & 3.03 & 1.07 & 1.00 & 2.30 & 1.56 & 1.32 & 1.01 & 0.63 & 0.78 & 3.17 & 0.59 & 4.54 & 0.38 & 0.18 \\
\hline FS17 & $\mathrm{H} 7$ & $3516.6-3521.0$ & 43 & 21.56 & 6.31 & 4.63 & 10.37 & 4.48 & 3.27 & 2.45 & 1.25 & 1.15 & 5.67 & n.d. & 3.98 & 0.19 & 0.50 \\
\hline FS18 & $\mathrm{H} 7$ & $3383.2-3493.4$ & 55 & 55.36 & 19.18 & 12.24 & 28.30 & 10.28 & 6.04 & 3.77 & 1.59 & 1.35 & 19.00 & n.d. & 3.98 & 0.17 & 1.30 \\
\hline FS19 & HD1 & $3329.4-3372.8$ & 56 & 14.57 & 3.46 & 2.48 & 5.83 & 3.61 & 2.59 & 1.95 & 1.07 & 1.04 & 7.33 & 0.61 & 3.48 & 0.68 & 1.25 \\
\hline FS20 & HD1-1 & $3347.0-3384.0$ & 45 & 12.90 & 4.82 & 3.24 & 7.71 & 3.28 & 2.22 & 2.00 & 1.06 & 1.04 & 1.78 & 0.57 & 3.24 & 0.86 & 1.09 \\
\hline FS21 & HD2 & $2542.0-2563.0$ & 40 & 5.96 & 2.15 & 1.64 & 3.79 & 2.06 & 1.68 & 1.34 & 0.78 & 0.87 & 4.24 & 0.61 & 4.22 & 0.47 & 0.31 \\
\hline FS22 & M1 & $3105.2-3108.0$ & 38 & 3.42 & 1.14 & 1.06 & 2.27 & 1.63 & 1.39 & 0.96 & 0.62 & 0.77 & 1.25 & 0.59 & 3.45 & 0.41 & 0.11 \\
\hline FS23 & M1 & $3084.0-3086.0$ & 37 & 3.32 & 1.03 & 0.98 & 2.38 & 1.39 & 1.33 & 0.98 & 0.65 & 0.79 & 1.37 & 0.59 & 3.45 & 0.46 & 0.11 \\
\hline FS24 & M2 & $3011.8-3047.6$ & 39 & 3.78 & 1.29 & 1.25 & 2.86 & 1.45 & 1.41 & 0.98 & 0.65 & 0.79 & 1.40 & 0.59 & 3.68 & 0.39 & 0.11 \\
\hline FS25 & JFn1 & $2213.5-2216.0$ & 38 & 4.49 & 1.29 & 1.17 & 2.68 & 2.02 & 1.76 & 1.26 & 0.78 & 0.87 & 1.84 & 0.59 & 4.71 & 0.53 & 0.32 \\
\hline FS26 & $\mathrm{H} 10$ & $2961.6-2981.8$ & 56 & 7.99 & 1.78 & 1.54 & 3.10 & 2.29 & 2.23 & 1.51 & 0.96 & 0.99 & 2.57 & 0.59 & 4.06 & 0.46 & 0.32 \\
\hline FS27 & $\mathrm{H} 2-13$ & $3043.4-3085.4$ & 46 & 4.46 & 1.39 & 1.44 & 3.13 & 2.02 & 1.94 & 1.17 & 0.80 & 0.92 & 2.33 & 0.59 & 4.28 & 0.28 & 0.26 \\
\hline FS28 & $\mathrm{H} 2-16$ & $2892.6-2972.0$ & 45 & 4.78 & 1.49 & 1.49 & 3.01 & 1.99 & 2.03 & 1.26 & 0.87 & 0.95 & 2.85 & 0.58 & 4.24 & 0.42 & 0.30 \\
\hline FS29 & H2-17X & 2998.6-3020.0 & 52 & 7.17 & 2.23 & 1.87 & 4.42 & 2.66 & 2.51 & 1.56 & 1.00 & 1.00 & 4.26 & 0.53 & 4.00 & 0.57 & 0.45 \\
\hline FS30 & $\mathrm{H} 2-18$ & $3151.6-3160.0$ & n.d. & 6.74 & 1.67 & 1.37 & 3.67 & 1.87 & 1.86 & 1.21 & 0.82 & 0.93 & 1.56 & 0.46 & 4.49 & 0.60 & 0.35 \\
\hline
\end{tabular}




\begin{tabular}{|c|c|c|c|c|c|c|c|c|c|c|c|c|c|c|c|c|c|}
\hline $\begin{array}{c}\text { Sample } \\
\text { No. }\end{array}$ & Well & Depth, m & API, ${ }^{\circ}$ & $4-/ 1-$ & $\begin{array}{l}4,6-/ \\
1,4-\end{array}$ & $\begin{array}{c}2,4-/ \\
1,4-\end{array}$ & $\begin{array}{c}(2,6+3,6) / \\
1,4-\end{array}$ & TMDBT-I1 & TMDBT-I2 & MPR & MPI-1 & $\begin{array}{l}R_{\mathrm{c}} \\
\%\end{array}$ & $\begin{array}{l}\mathrm{Ts} / \\
\mathrm{Tm}\end{array}$ & $\begin{array}{c}22 \mathrm{~S} / \\
(22 \mathrm{~S}+22 \mathrm{R})\end{array}$ & $\begin{array}{l}\mathrm{Pr} / \\
\mathrm{Ph}\end{array}$ & $\begin{array}{l}\text { ADBT/ } \\
\text { ADBF }\end{array}$ & $\begin{array}{c}\text { Oleanane/ } \\
\mathrm{C}_{30} \mathrm{H}\end{array}$ \\
\hline FS31 & $\mathrm{H} 2-5$ & $3114.0-3148.8$ & 59 & 6.32 & 1.77 & 1.52 & 3.20 & 2.51 & 2.25 & 1.35 & 0.89 & 0.96 & 1.08 & 0.54 & 4.27 & 1.04 & 0.17 \\
\hline FS32 & $\mathrm{H} 2-6$ & $3147.8-3192.0$ & 52 & 6.89 & 1.83 & 1.60 & 3.52 & 2.34 & 2.20 & 1.31 & 0.90 & 0.96 & 2.45 & 0.50 & 4.19 & 0.56 & 0.33 \\
\hline FS33 & $H 3-12 x$ & 3194.4-3238.8 & 47 & 5.38 & 1.37 & 1.27 & 3.38 & 1.77 & 1.80 & 1.14 & 0.78 & 0.91 & 3.55 & 0.51 & 3.98 & 0.55 & 0.30 \\
\hline FS34 & H3-5 & $3109.1-3128.2$ & 54 & 5.44 & 1.53 & 1.41 & 3.49 & 1.74 & 1.65 & 1.29 & 0.84 & 0.94 & 1.86 & 0.59 & 4.31 & 0.55 & 0.20 \\
\hline FS35 & H3-6 & $3114.4-3148.2$ & 52 & 5.57 & 1.56 & 1.47 & 3.51 & 1.99 & 1.95 & 1.25 & 0.85 & 0.94 & 3.00 & 0.59 & 3.98 & 0.47 & 0.29 \\
\hline FS36 & $\mathrm{H} 4-3$ & $3015.4-3060.6$ & 39 & 4.50 & 1.12 & 1.08 & 2.77 & 1.90 & 1.64 & 1.15 & 0.76 & 0.90 & 1.94 & 0.58 & 4.58 & 0.31 & 0.27 \\
\hline FS37 & H8-1 & $3585.2-3669.2$ & 48 & 11.34 & 2.81 & 2.39 & 4.66 & 3.25 & 2.68 & 1.73 & 1.15 & 1.06 & 6.14 & 0.32 & 3.36 & 1.23 & 1.07 \\
\hline FS38 & H8-1X & $3678.0-3733.6$ & n.d. & 13.27 & 3.48 & 2.70 & 6.87 & 2.92 & 2.52 & 2.10 & 1.26 & 1.11 & n.d. & n.d. & 3.19 & 1.75 & n.d. \\
\hline FS39 & HD1-5 & $3486.2-3510.4$ & 59 & 9.83 & 2.63 & 2.25 & 5.15 & 2.72 & 2.66 & 1.80 & 1.09 & 1.04 & 1.33 & 0.51 & 4.34 & 1.21 & 0.24 \\
\hline FS40 & HD1-7 & $3450.7-3462.5$ & n.d. & 9.69 & 2.55 & 2.08 & 5.01 & 2.51 & 2.20 & 1.77 & 1.07 & 1.03 & 1.33 & 0.50 & 4.18 & 1.36 & 0.25 \\
\hline FS41 & Hong3 & $2504.0-2513.4$ & n.d. & 4.84 & 1.23 & 1.23 & 2.43 & 1.51 & 1.57 & 1.10 & 0.81 & 0.92 & 1.38 & 0.52 & 4.64 & 0.50 & 0.23 \\
\hline FS42 & Hong5 & $2504.0-2513.4$ & n.d. & 3.35 & 0.99 & 1.10 & 2.62 & 1.65 & 1.77 & 1.06 & 0.76 & 0.90 & 2.33 & 0.60 & 4.35 & 0.28 & 0.29 \\
\hline FS43 & Hong5-1 & $2725.4-2775.0$ & n.d. & 2.01 & 0.60 & 0.76 & 2.05 & 0.90 & 1.02 & 0.91 & 0.65 & 0.86 & 1.56 & 0.55 & 4.38 & 0.26 & 0.93 \\
\hline FS44 & L11 & $2842.4-2846.0$ & 42 & 5.21 & 1.03 & 1.36 & 2.42 & 1.57 & 1.67 & 1.51 & 0.71 & 0.88 & 1.22 & 0.55 & 4.95 & 0.16 & 0.62 \\
\hline FS45 & L12 & $2794.8-2801.0$ & 32 & 5.20 & 0.87 & 2.02 & 2.86 & 2.28 & 1.99 & 1.90 & 0.70 & 0.88 & 1.27 & 0.55 & 5.12 & 0.16 & 0.81 \\
\hline FS46 & M4 & $3208.2-3275.8$ & 36 & 2.77 & 1.02 & 0.96 & 2.50 & 1.14 & 1.17 & 1.00 & 0.64 & 0.85 & 1.27 & 0.57 & 3.70 & 0.38 & 0.15 \\
\hline FS47 & M4-1 & $3459.6-3493.4$ & 39 & 2.94 & 0.82 & 0.89 & 1.89 & 1.05 & 1.04 & 0.86 & 0.61 & 0.84 & 1.38 & 0.52 & 3.99 & 0.39 & 0.18 \\
\hline FS48 & M5 & $3217.3-3219.0$ & n.d. & 3.47 & 0.97 & 1.04 & 2.82 & 1.30 & 1.35 & 0.98 & 0.69 & 0.87 & 1.50 & 0.52 & 4.06 & 0.54 & 0.16 \\
\hline FS49 & M5-1 & $3150.0-3160.1$ & n.d. & 3.88 & 1.01 & 1.00 & 2.57 & 1.31 & 1.40 & 0.97 & 0.67 & 0.87 & 1.63 & 0.56 & 4.07 & 0.45 & 0.16 \\
\hline FS50 & M6X & $2628.2-2631.0$ & n.d. & 3.68 & 1.03 & 1.03 & 2.32 & 1.29 & 1.40 & 0.94 & 0.68 & 0.87 & 1.38 & 0.52 & 4.24 & 0.47 & 0.19 \\
\hline
\end{tabular}

Notes: 4-/1-: 4-/1-methyldibenzothiophene; 4,6-/1,4-: 4,6-/1,4-dimethyldibenzothiophene; 2,4-/1,4-: 2,4-/1,4-dimethyldibenzothiophene; (2,6 + 3,6)-/1,4-: (2,6 + 3,6)-/1,4-dimethyldibenzothiophene; TMDBT-I1: 2,4,6-/(1,4,6 + 1,4,8 + 3,4,6)-TMDBT; TMDBT-I2: $(2,4,8+2,4,7)-/(1,4,6+$ 1,4,8 + 3,4,6)-TMDBT; MPR: 2-/1-MP (MP: methylphenanthrene); MPI-1: $1.5 \times(2-\mathrm{MP}+3-\mathrm{MP}) /(\mathrm{Phen}+9-\mathrm{MP}+1-\mathrm{MP})\left(\right.$ Phen: phenanthrene); $R_{\mathrm{c}}$ $(\%)=0.4+0.6 \times \mathrm{MPI}-1 ; 22 \mathrm{~S} /(22 \mathrm{~S}+22 \mathrm{R}): \mathrm{C}_{31}$ hopane $22 \mathrm{~S} /(22 \mathrm{~S}+22 \mathrm{R}) ; \mathrm{Pr} / \mathrm{Ph}$ : Pristane/Phytane; ADBT/ADBF: alkylated dibenzothiophene/alkylated dibenzofuran; Oleanane $/ \mathrm{C}_{30} \mathrm{H}$ : oleanane $/ \mathrm{C}_{30}$ hopane

All oils are characterized by extremely high $\mathrm{Pr} / \mathrm{Ph}$ ratios (with an average of 4.01), high abundance of oleananes (Table 1). The abundance of oleananes is likely to be sensitive to changes in Eh and $\mathrm{pH}$ during early diagenesis (Murray et al, 1997), but the extremely high oleanane abundances in oils and sediment extracts are usually related to the organic matter input from angiosperm higher land plants (Moldowan et al, 1994; Li et al, 2008c). In the cross plot of $\mathrm{Pr} / \mathrm{Ph}-\mathrm{ADBT} /$ ADBF (Radke et al, 2000), all data points of the oils and condensates from the Fushan Depression fall into the Zone 4, suggesting their terrestrial/deltaic origin.

A total of 45 oils were selected from the Tahe oil field, Tarim Basin, NW China. All the oils mainly occur in the Ordovician carbonates and Triassic sandstones. The majority of the discovered oil accumulations originated from the Middle-Upper Ordovician marine carbonate source rocks (Zhang and Huang, 2005; Wang et al, 2008; Cui et al, 2013). They are characterized by low $\mathrm{Pr} / \mathrm{Ph}$ and high gammacerane/ $\mathrm{C}_{30}$ hopane ratios (Table 2), which indicate anoxic and saline depositional environments of the source rocks. The higher $\mathrm{C}_{29} / \mathrm{C}_{30}$ hopane (>0.6) and $\mathrm{C}_{35} / \mathrm{C}_{34}$ hopane (>0.8) ratios (Table
2) suggest their marine carbonate origin (Peters et al, 2005). All oils collected from the Tahe oil field fall into Zone 1A of the cross plot of $\mathrm{Pr} / \mathrm{Ph}-\mathrm{ADBT} / \mathrm{ADBF}$ (Fig. 2), confirming their marine carbonate origin.

Some oils in the Tahe oil field have undergone severe biodegradation (Wang et al, 2008). The distribution of PAHs may be influenced at advanced stages of biodegradation. For example, with more severe microbial attack, methylphenanthrene isomers of the $\alpha$-type are more refractory than $\beta$-type isomers (Rowland et al, 1986; Radke, 1988). Bao and Zhu (2009) also reported that the relative abundances and distribution patterns may be influenced by secondary alteration processes, such as water washing and biodegradation. Because this study mainly focuses on the DBT related maturity parameters, those samples which have potential biodegradation signatures, such as very low $\mathrm{API}^{\circ}$, extremely high $\mathrm{Pr} / n \mathrm{C}_{17}$ and $\mathrm{Ph} / n \mathrm{C}_{18}$ ratios and apparent unresolved complex mixture (UCM) baseline humps of the gas chromatograms are therefore excluded and biodegradation is not considered in the following discussion.

Forty-five oil samples collected from the Termit Basin, 
Table 2 Methylated dibenzothiophenes related maturity parameters in oils from the Tarim Basin, NW China

\begin{tabular}{|c|c|c|c|c|c|c|c|c|c|c|c|c|c|c|c|c|c|c|}
\hline $\begin{array}{c}\text { Sample } \\
\text { No. }\end{array}$ & Well & Formation & API, ${ }^{\circ}$ & $4-/ 1-$ & $\begin{array}{l}4,6- \\
/ 1,4- \\
\end{array}$ & $\begin{array}{l}2,4- \\
11,4- \\
\end{array}$ & $\begin{array}{c}(2,6+3,6)- \\
\quad 11,4-\end{array}$ & TMDBT-I1 & TMDBT-I2 & MPR & MPI-1 & $R_{\mathrm{c}}, \%$ & $\begin{array}{l}\text { Ts/ } \\
\text { Tm }\end{array}$ & $\begin{array}{c}22 \mathrm{~S} / \\
(22 \mathrm{~S}+22 \mathrm{R})\end{array}$ & $\mathrm{Pr} / \mathrm{Ph}$ & $\begin{array}{l}\mathrm{ADBT} / \\
\mathrm{ADBF}\end{array}$ & $\begin{array}{l}\mathrm{C}_{29} / \mathrm{C}_{30} \\
\text { hopane }\end{array}$ & $\begin{array}{l}\mathrm{C}_{35} / \mathrm{C}_{34} \\
\text { hopane }\end{array}$ \\
\hline TH01 & S1066 & $\mathrm{O}_{3} \mathrm{q}-\mathrm{O}_{2} \mathrm{yj}$ & 26 & 1.97 & 1.02 & 0.51 & 1.40 & 1.92 & 1.46 & 0.79 & 0.55 & 0.82 & 0.54 & 0.61 & 0.74 & 5.66 & 0.74 & 0.69 \\
\hline TH02 & S7202 & $\mathrm{O}_{2} \mathrm{yj}$ & 31 & 2.88 & 1.46 & 0.66 & 2.28 & 2.41 & 1.66 & 0.82 & 0.57 & 0.83 & 0.52 & 0.62 & 0.73 & 6.76 & 0.78 & 0.92 \\
\hline TH03 & TK1010 & $\mathrm{O}_{2} \mathrm{yj}$ & 26 & 2.92 & 1.48 & 0.66 & 2.13 & 2.27 & 1.69 & 0.80 & 0.57 & 0.83 & 0.56 & 0.61 & 0.81 & 7.40 & 0.90 & 0.89 \\
\hline TH04 & TK241 & $\mathrm{O}_{2} \mathrm{yj}$ & 19 & 3.03 & 1.68 & 0.75 & 2.09 & 2.22 & 1.66 & 0.87 & 0.58 & 0.83 & 0.47 & 0.61 & 0.72 & 6.35 & 0.88 & 1.10 \\
\hline TH05 & S108 & $\mathrm{O}_{2} \mathrm{yj}$ & 33 & 3.12 & 1.07 & 0.53 & 1.77 & 1.98 & 1.51 & 0.78 & 0.55 & 0.82 & 0.67 & 0.60 & 0.68 & 7.60 & 0.69 & 0.80 \\
\hline TH06 & TK207 & $\mathrm{C}_{1} \mathrm{~b}-\mathrm{O}_{1-2} \mathrm{y}$ & 15 & 3.24 & 1.53 & 0.74 & 1.79 & 2.47 & 1.65 & 0.81 & 0.55 & 0.82 & 0.39 & 0.59 & 0.80 & 9.40 & 0.91 & 1.05 \\
\hline TH07 & S116-1 & $\mathrm{O}_{3} \mathrm{q}-\mathrm{O}_{2} \mathrm{yj}$ & 29 & 3.37 & 1.69 & 0.76 & 2.42 & 2.56 & 1.83 & 0.86 & 0.62 & 0.85 & 0.54 & 0.61 & 0.72 & 7.44 & 0.80 & 0.80 \\
\hline TH08 & S106-1 & $\mathrm{O}_{3} \mathrm{q}-\mathrm{O}_{2} \mathrm{yj}$ & 34 & 3.45 & 1.79 & 0.72 & 2.13 & 2.57 & 1.77 & 0.68 & 0.49 & 0.80 & 0.75 & 0.63 & 0.83 & 9.80 & 0.75 & 0.80 \\
\hline TH09 & S116-2 & $\mathrm{O}_{3} \mathrm{q}-\mathrm{O}_{2} \mathrm{yj}$ & 37 & 3.69 & 1.84 & 0.76 & 2.48 & 2.64 & 1.73 & 0.81 & 0.55 & 0.82 & 0.89 & 0.63 & 0.67 & 7.75 & 0.65 & 0.88 \\
\hline TH10 & S1181 & $\mathrm{O}_{3} \mathrm{q}-\mathrm{O}_{2} \mathrm{yj}$ & 38 & 3.89 & 1.92 & 0.82 & 2.85 & 2.86 & 1.93 & 0.84 & 0.59 & 0.84 & 0.82 & 0.63 & 0.77 & 8.06 & 0.71 & 0.95 \\
\hline TH11 & S106-3 & $\mathrm{O}_{3} \mathrm{q}-\mathrm{O}_{2} \mathrm{yj}$ & 38 & 4.09 & 2.06 & 0.86 & 2.87 & 2.96 & 2.03 & 0.80 & 0.58 & 0.83 & 1.04 & 0.63 & 0.82 & 8.60 & 0.74 & 0.86 \\
\hline TH12 & S106 & $\mathrm{O}_{3} \mathrm{q}-\mathrm{O}_{2} \mathrm{yj}$ & 39 & 4.24 & 2.16 & 0.87 & 2.74 & 2.44 & 1.59 & 0.69 & 0.51 & 0.81 & 1.22 & 0.62 & 0.84 & 13.45 & 0.77 & 1.00 \\
\hline TH13 & TK1109 & $\mathrm{O}_{2} \mathrm{yj}$ & 40 & 4.28 & 2.13 & 0.90 & 3.07 & 3.20 & 2.17 & 0.82 & 0.59 & 0.84 & 1.00 & 0.63 & 0.88 & 7.74 & 0.70 & 0.73 \\
\hline TH14 & TK1108 & $\mathrm{O}_{2} \mathrm{yj}$ & 39 & 4.30 & 2.09 & 0.89 & 2.94 & 3.27 & 2.16 & 0.79 & 0.58 & 0.83 & 1.00 & 0.63 & 0.77 & 8.85 & 0.69 & 0.62 \\
\hline TH15 & S117 & $\mathrm{O}_{3} 1-\mathrm{O}_{2} \mathrm{yj}$ & 38 & 4.46 & 2.09 & 0.87 & 3.04 & 3.07 & 2.08 & 0.80 & 0.58 & 0.83 & 1.63 & 0.65 & 0.70 & 8.45 & 0.70 & 0.67 \\
\hline TH16 & T901 & $\mathrm{O}_{2} \mathrm{yj}$ & 34 & 4.84 & 2.23 & 0.95 & 2.82 & 3.34 & 2.05 & 1.03 & 0.69 & 0.88 & 0.52 & 0.60 & 0.91 & 8.86 & 0.91 & 1.14 \\
\hline TH17 & S7203 & $\mathrm{O}_{3} \mathrm{q}-\mathrm{O}_{2} \mathrm{yj}$ & 38 & 5.43 & 2.65 & 1.06 & 3.44 & 3.61 & 2.36 & 1.00 & 0.70 & 0.88 & 1.00 & 0.66 & 0.90 & 10.08 & 0.70 & 0.98 \\
\hline TH18 & LN44 & $\mathrm{O}$ & n.d. & 5.57 & 2.77 & 1.12 & 3.33 & 3.65 & 2.36 & 1.04 & 0.72 & 0.89 & 1.50 & 0.66 & 0.70 & 9.67 & 0.72 & n.d. \\
\hline TH19 & LN51 & $\mathrm{O}$ & n.d. & 6.12 & 3.27 & 1.44 & 3.40 & 4.33 & 2.79 & 1.20 & 0.82 & 0.93 & 1.94 & 0.66 & 0.83 & 10.21 & 0.45 & n.d. \\
\hline TH20 & T208 & $\mathrm{O}_{3} \mathrm{l}-\mathrm{O}_{3} \mathrm{q}$ & 18 & 6.42 & 3.22 & 1.30 & 3.82 & 3.90 & 2.50 & 1.08 & 0.73 & 0.89 & 0.92 & 0.65 & 0.65 & 9.25 & 0.83 & 0.83 \\
\hline TH21 & S7201 & $\mathrm{O}_{3} 1-\mathrm{O}_{2} \mathrm{yj}$ & 42 & 6.60 & 3.51 & 1.52 & 4.21 & 4.99 & 2.99 & 1.61 & 1.02 & 1.01 & 0.49 & 0.62 & 0.76 & 11.89 & 0.95 & 1.00 \\
\hline TH22 & S112-1 & $\mathrm{O}_{3} \mathrm{~s}-\mathrm{O}_{2} \mathrm{yj}$ & 39 & 6.64 & 3.32 & 1.33 & 4.20 & 4.22 & 2.67 & 0.99 & 0.72 & 0.89 & 1.86 & 0.68 & 0.63 & 9.92 & 0.66 & 0.74 \\
\hline TH23 & T913 & $\mathrm{O}_{3} \mathrm{~s}-\mathrm{O}_{2} \mathrm{yj}$ & 38 & 7.41 & 3.05 & 1.34 & 3.88 & 4.08 & 2.69 & 1.10 & 0.75 & 0.90 & 4.56 & 0.70 & 0.87 & 8.68 & 0.71 & n.d. \\
\hline TH24 & LN54 & $\mathrm{O}$ & n.d. & 7.49 & 3.11 & 1.36 & 4.13 & 4.53 & 2.81 & 1.17 & 0.78 & 0.91 & 2.33 & 0.70 & 0.71 & 9.09 & 0.69 & n.d. \\
\hline TH25 & LG12 & $\mathrm{O}_{1-2} \mathrm{y}$ & n.d. & 7.98 & 3.49 & 1.54 & 4.24 & 4.61 & 3.08 & 1.03 & 0.79 & 0.91 & 4.26 & 0.70 & 0.85 & 12.12 & 0.74 & n.d. \\
\hline TH26 & S112 & $\mathrm{O}_{2} \mathrm{yj}$ & 41 & 8.05 & 3.48 & 1.32 & 4.37 & 4.49 & 2.74 & 0.88 & 0.66 & 0.86 & 4.88 & 0.78 & 0.68 & 9.47 & 0.71 & n.d. \\
\hline TH27 & LG13 & $\mathrm{O}$ & n.d. & 10.75 & 4.28 & 1.93 & 5.45 & 5.98 & 3.73 & 1.39 & 0.85 & 0.94 & 6.14 & 0.72 & 0.79 & 9.75 & 0.68 & n.d. \\
\hline TH28 & TK925H & $\mathrm{T}_{2} \mathrm{a}^{1}$ & 23 & 3.96 & 2.13 & 0.87 & 2.13 & 2.90 & 1.68 & 0.79 & 0.55 & 0.82 & 0.50 & 0.61 & 0.86 & 15.65 & 0.90 & 0.89 \\
\hline TH29 & TK926H & $\mathrm{T}_{2} \mathrm{a}$ & 24 & 4.19 & 2.13 & 0.94 & 2.55 & 3.06 & 2.00 & 0.90 & 0.62 & 0.85 & 0.46 & 0.62 & 0.86 & 8.34 & 0.92 & 1.35 \\
\hline TH30 & TK933H & $\mathrm{T}_{2} \mathrm{a}^{1}$ & 54 & 4.27 & 2.24 & 0.95 & 2.40 & 3.05 & 1.89 & 0.92 & 0.60 & 0.84 & 0.48 & 0.61 & 0.89 & 10.64 & 0.85 & 1.08 \\
\hline TH31 & TK910H & $\mathrm{T}_{2} \mathrm{a}^{1}$ & 23 & 4.32 & 2.17 & 0.95 & 2.55 & 2.97 & 2.03 & 0.89 & 0.61 & 0.84 & 0.50 & 0.62 & 0.78 & 7.76 & 0.91 & 1.21 \\
\hline TH32 & TK909H & $\mathrm{T}_{2} \mathrm{a}^{1}$ & 22 & 4.40 & 2.15 & 0.93 & 2.57 & 3.11 & 2.10 & 0.92 & 0.63 & 0.85 & 0.45 & 0.63 & 0.87 & 8.64 & 0.93 & 0.95 \\
\hline TH33 & LN51 & $\mathrm{T}$ & n.d. & 4.43 & 2.30 & 1.03 & 2.58 & 3.07 & 1.98 & 0.91 & 0.64 & 0.85 & 0.63 & 0.61 & 0.97 & 7.26 & 0.92 & 0.86 \\
\hline TH34 & T901 & $\mathrm{T}_{2} \mathrm{a}^{1}$ & 34 & 4.51 & 2.28 & 0.98 & 2.82 & 3.34 & 2.05 & 1.03 & 0.69 & 0.88 & 0.51 & 0.63 & 0.81 & 8.86 & 0.92 & 0.98 \\
\hline TH35 & TK932H & $\mathrm{T}_{2} \mathrm{a}^{1}$ & 52 & 4.63 & 2.37 & 1.01 & 2.83 & 3.47 & 2.24 & 1.02 & 0.69 & 0.88 & 0.61 & 0.63 & 0.90 & 9.16 & 0.90 & 0.87 \\
\hline TH36 & TK927H & $\mathrm{T}_{2} \mathrm{a}^{1}$ & 46 & 4.73 & 2.17 & 0.99 & 2.88 & 3.10 & 2.12 & 1.09 & 0.70 & 0.88 & 0.51 & 0.62 & 0.86 & 7.83 & 0.88 & 1.18 \\
\hline TH37 & TK919H & $\mathrm{T}_{2} \mathrm{a}^{1}$ & 37 & 4.78 & 2.35 & 1.00 & 2.83 & 3.13 & 2.04 & 1.00 & 0.68 & 0.87 & 0.52 & 0.62 & 0.72 & 8.66 & 0.91 & 1.00 \\
\hline TH38 & TK920H & $\mathrm{T}_{2} \mathrm{a}^{1}$ & 34 & 4.82 & 2.37 & 0.98 & 2.87 & 3.57 & 2.06 & 1.08 & 0.66 & 0.86 & 0.50 & 0.62 & 0.93 & 11.30 & 0.97 & 0.86 \\
\hline TH39 & S112-2 & $\mathrm{S}_{1} \mathrm{k}$ & 38 & 4.93 & 2.42 & 0.92 & 2.66 & 3.38 & 2.01 & 0.83 & 0.50 & 0.80 & 1.10 & 0.71 & 0.87 & 7.66 & 0.78 & n.d. \\
\hline TH40 & THN3 & $\mathrm{T}_{2} \mathrm{a}^{3}$ & 42 & 5.54 & 2.97 & 1.16 & 3.23 & 4.14 & 2.41 & 0.99 & 0.72 & 0.89 & 0.86 & 0.65 & 0.72 & 6.28 & 0.87 & 1.09 \\
\hline TH41 & THN8 & $\mathrm{T}_{2} \mathrm{a}^{3}$ & 60 & 6.13 & 3.41 & 1.12 & 3.12 & 4.36 & 2.10 & 1.05 & 0.70 & 0.88 & 1.12 & 0.65 & 0.93 & 2.94 & 0.97 & n.d. \\
\hline TH42 & THN9H & $\mathrm{T}_{2} \mathrm{a}^{3}$ & 54 & 6.40 & 3.34 & 1.17 & 3.29 & 3.88 & 2.03 & 1.04 & 0.70 & 0.88 & 1.00 & 0.66 & 0.79 & 3.78 & 0.82 & 0.73 \\
\hline TH43 & LN44 & $\mathrm{T}$ & n.d. & 6.49 & 3.06 & 1.21 & 3.57 & 3.58 & 2.09 & 1.15 & 0.78 & 0.91 & 1.09 & 0.61 & 1.00 & n.d. & 0.93 & 0.86 \\
\hline TH44 & THN2 & $\mathrm{T}_{2} \mathrm{a}^{3}$ & 54 & 6.62 & 3.28 & 1.20 & 3.54 & 3.91 & 2.20 & 1.15 & 0.72 & 0.89 & 0.84 & 0.68 & 0.81 & 4.76 & 0.78 & n.d. \\
\hline TH45 & THN5H & $\mathrm{T}_{2} \mathrm{a}^{3}$ & 57 & 7.10 & 3.44 & 1.14 & 3.41 & 4.17 & 2.11 & 1.13 & 0.70 & 0.88 & 1.04 & 0.68 & 0.84 & 3.84 & 0.84 & n.d. \\
\hline
\end{tabular}


Eastern Nigeria (Africa) mainly occur in Eocene sandstones and originated from Mesozoic marine shales. They are characterized by relatively low $\mathrm{Pr} / \mathrm{Ph}$ and high gammacerane/ $\mathrm{C}_{30}$ hopane ratios (Wan et al, 2014). In the cross plot of the $\mathrm{Pr} / \mathrm{Ph}-\mathrm{ADBT} / \mathrm{ADBF}$ (Fig. 2), most of the oil samples fall into the marine shale zone.

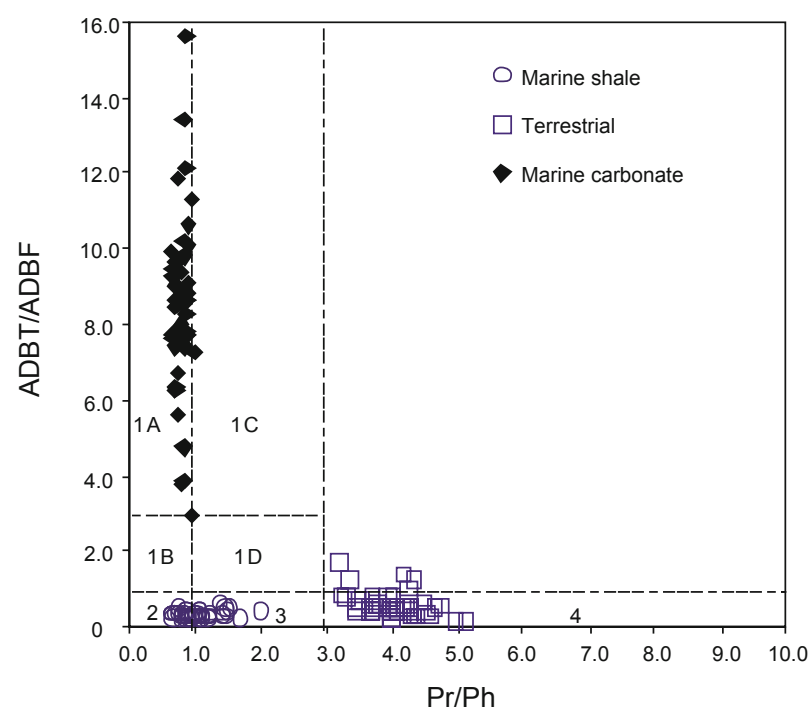

Fig. 2 Concentration ratios of ADBT/ADBF (alkylated dibenzothiophene / alkylated dibenzofuran) plotted against $\mathrm{Pr} / \mathrm{Ph}$ (pristane/phytane) for marine shale (०) from Termit Basin, Eastern Nigeria (Africa), terrestrial shale ( $\square$ ) from Beibuwan Basin, South China Sea, and marine carbonate $(\bullet)$ from the Tarim Basin. Zones indicated by broken lines are used for interpreting depositional environments. Zone 1A: Marine carbonate; Zone 1B: Marine carbonate, marine marl and lacustrine sulfate-rich; Zones 1C and 1D: Mature mudstones and high-rank coals deposited in lacustrine swamp environments with possible marine incursions; Zone 2: Lacustrine sulfate-poor rocks of variable lithology; Zone 3: Marine and lacustrine shales; Zone 4: Fluvial/ deltaic carbonaceous shales and coals (Radke et al, 2000)

\subsection{Laboratory geochemical analyses}

All oils and condensates were deasphaltened using $n$-hexane and then fractionated by liquid chromatography using silica gel/alumina columns into saturated and aromatic hydrocarbon fractions using $n$-hexane with dichloromethane $(50: 50, \mathrm{v}: \mathrm{v})$ and dichloromethane as respective eluents.

The GC-MS analyses of the aromatic hydrocarbon fractions and mixture solutions were performed on an Agilent 5975i GC-MS system equipped with an HP-5 MS (5\%-phenyl-methylpolysiloxane) fused silica capillary column (60 $\mathrm{m} \times 0.25 \mathrm{~mm}$ i.d., with a $0.25 \mu \mathrm{m}$ film thickness). The GC operating conditions were as follows: the temperature was held initially at $80{ }^{\circ} \mathrm{C}$ for $1 \mathrm{~min}$, ramped to $310^{\circ} \mathrm{C}$ at 3 ${ }^{\circ} \mathrm{C} / \mathrm{min}$, and then kept isothermal for $16 \mathrm{~min}$. Helium was used as the carrier gas. The injector temperature was set to $300{ }^{\circ} \mathrm{C}$. The MS was operated in the electron impact (EI) mode with an ionization energy of $70 \mathrm{eV}$, and a scan range of 50-600 Da.

\subsection{Identification of isomers of methylated dibenzothiophenes}

Two MDBT isomers MDBTs, i.e. 1-MDBT and 4-MDBT, were identified on $\mathrm{m} / \mathrm{z} 198$ mass chromatograms
(Connan et al, 1986). Peak identifications for MDBTs in this study were performed by comparison with published relative GC retention time and retention indices (Lee et al, 1979; Vassilaros et al, 1982). Three DMDBT isomers, i.e. 1,4-DMDBT, 2,4-DMDBT and 4,6-DMDBT were identified by co-injection of authentic standards (Budzinski et al, 1991; Chakhmakhchev et al, 1997). A recent study shows that the 1,6-DMDBT isomer may co-elute with 1,4-DMDBT ( $\mathrm{Li}$ et al, 2012). However, 1,4-DMDBT and 1,6-DMDBT have similar thermodynamic stability (Richard, 2001). Thus, the co-elution of 1,6-DMDBT and 1,4- has no significant influence on the application of the 4,6-/1,4-DMDBT or 2,4-/1,4-DMDBT maturity parameters. By comparison of relative retention times with literature (Asif, 2010) and calculated retention indices (Schade and Andersson, 2006), other DMDBT isomers including 2,6-, 3,6-DMDBT, can be firmly identified in $\mathrm{m} / \mathrm{z} 212$ mass chromatograms (Fig. 1).

Five trimethyldibenzothiophenes (TMDBTs) have been tentatively detected on $\mathrm{m} / \mathrm{z} 226$ mass chromatograms (Chakhmakhchev et al, 1997). Their chemical structures, i.e. the specific positions of the three methyl groups, however, have not yet been assigned. Schade and Andersson (2006) specified a series of alkylated dibenzothiophenes through correlation of structure and gas chromatographic retention indices. By correlation with the retention indexes $\left(I_{\mathrm{S}}\right.$ and $I_{\mathrm{C}}$ ) proposed by Schade and Andersson (2006) and by comparison with co-elution of internal standards (Fig. 1) (2,4,7-TMDBT, 2,4,8-TMDBT, 4-E-6-MDBT were purchased from Chiron, Trondheim Norway; 1,3,7- and 1,4,7-DMDBT were purchased from Prof. Jan Andersson's Laboratory, Münster, Germany), a series of trimethyldibenzothiophene isomers were identified in the $m / z 226$ mass chromatograms in this study (Fig. 1).

\section{Results and discussion}

\subsection{Maturity parameters related to dimethyldibenzothiophenes}

MDR (4-/1-MDR ratio) proposed by Radke et al (1986) and Radke (1988), 4,6-/1,4-DMDBT and 2,4-/1,4-DMDBT ratios by Chakhmakhchev et al (1997), have been widely used in oil maturity assessments. The MDR has been calibrated against vitrinite reflectance $\left(R_{\mathrm{o}} \%\right)$, and empirical formulas for estimating vitrinite reflectance $\left(R_{\mathrm{c}} \%\right)$ have been introduced (Radke, 1988; Dzou et al, 1995; Luo et al, 2001). Two DMDBT based maturity parameters, i.e. 4,6-/1,4-DMDBT and 2,4-/1,4-DMDBT proposed by Chakhmakhchev and Suzuki (1995) and Chakhmakhchev et al (1997) also correlated well with other maturity indicators. All these DBT based maturity parameters are very useful for the characterization of very mature oils lacking biomarkers and work well over a wide range of catagenesis.

All these aforementioned maturity ratios have been calculated for 140 oil samples in this study to examine their interrelationship with each other (Tables 1, 2). For each data set, the 4,6-/1,4-DMDBT ratio correlates well with the MDR and 2,4-/1,4-DMDBT ratio (with correlation coefficients in the range of $R^{2}=0.68-0.93$ ) and have approximately the same trend (nearly parallel regression lines) (Fig. 3(a) and 3(b)), suggesting their similar behavior with increasing maturity. 

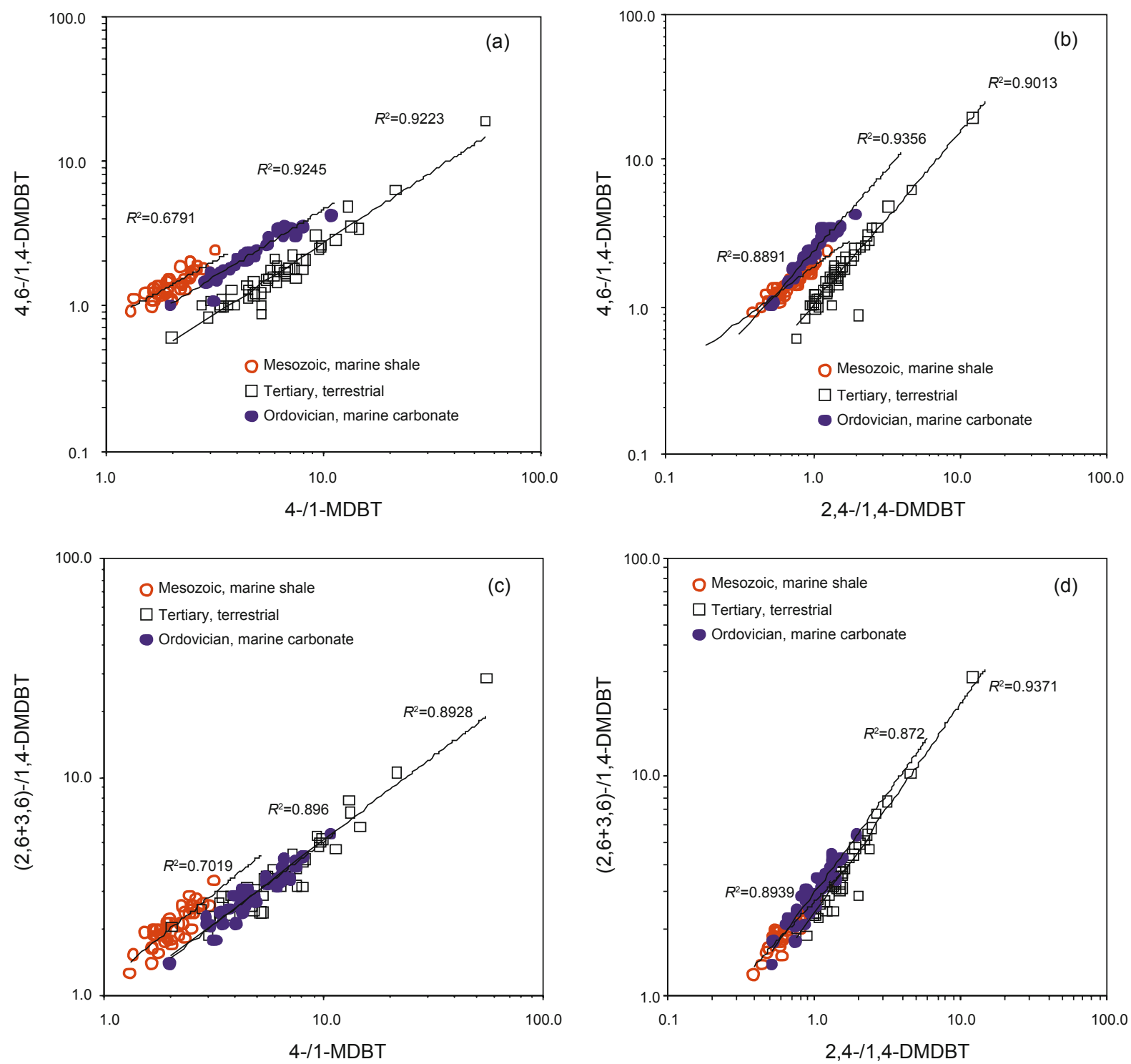

Fig. 3 Cross plots showing a good correlation between (a) 4,6-/1,4-DMDBT and 4-/1-MDBT ratios, (b) 4,6-/1,4-DMDBT and 2,4-/1,4-DMDBT ratios, (c) (2,6 + 3,6)-/1,4-DMDBT and 4-/1-MDBT ratios, and (d) (2,6 + 3,6)-/1,4-DMDBT and 2,4-/1,4-DMDBT ratios

In order to study the influence of organic facies on DBT based maturity parameters, some other maturity indicators must be chosen to serve as reference. Most of the sterane or terpane isomerization maturity parameters, such as $\mathrm{C}_{31}$ hopane $22 \mathrm{~S} /(22 \mathrm{~S}+22 \mathrm{R})$, however, are not good candidates due to their low concentrations or having reached their isomerization equilibrium point in high mature oils or condensates (Peters et al, 2005). Some aromatic hydrocarbon based parameters, such as methylphenanthrene index (MPI-1) show a strong linear correlation with increasing vitrinite reflectance and are often related to $R_{\mathrm{o}} \%$ for the principal phase of oil formation (Radke and Welte, 1981; Radke et al, 1982; Farrington et al, 1988; Radke, 1988; Radke et al, 1997). The empirical relationships derived from regression analysis (Radke and Welte, 1981; Radke, 1988) have been widely used in the calculation of equivalent vitrinite reflectance $\left(R_{\mathrm{c}} \%\right)$, especially for high mature oils or carbonate sequences without vitrinite of good quality. In this study, the $R_{\mathrm{c}}(\%)$ for all oils and condensates were calculated using the empirical relationship $R_{\mathrm{c}}(\%)=0.40$
$+0.6 \times$ MPI-1 (for $0.65 \%$ to $1.35 \% R_{\mathrm{m}}$ ) (Radke and Welte, 1981; Radke, 1988).

Fig. 4 illustrates the relationship between MDR (4-/1MDBT) and $R_{\mathrm{c}}(\%)$. The MDR exhibits an exponential relationship with $R_{\mathrm{c}}(\%)$. MDR increases slightly with maturity in the $0.7 \%-1.0 \%$ range and increases rapidly at advanced thermal stage $\left(R_{\mathrm{c}}=1.0 \%-1.35 \%\right)$, approximately consistent with previous observations (Schou and Myhr, 1988; Dzou et al, 1995). It is important to note that the oils and condensates in this study originated from three different types of source rocks yet have nearly same MDR relationship with $R_{\mathrm{c}}(\%)$. This suggests that lithologies and organic facies may have no significant influence on the 4-MDBT and 1-MDBT distributions in this maturity range. Although organic matter type and lithology of source rocks may affect the distributions of DBTs at early stages of thermal transformation (Radke et al, 1986; Connan et al, 1986; Huang and Pearson, 1999; Li et al, 2013b), the influence seems to reduce to a negligible level within the main oil window to post and over-mature stages. 


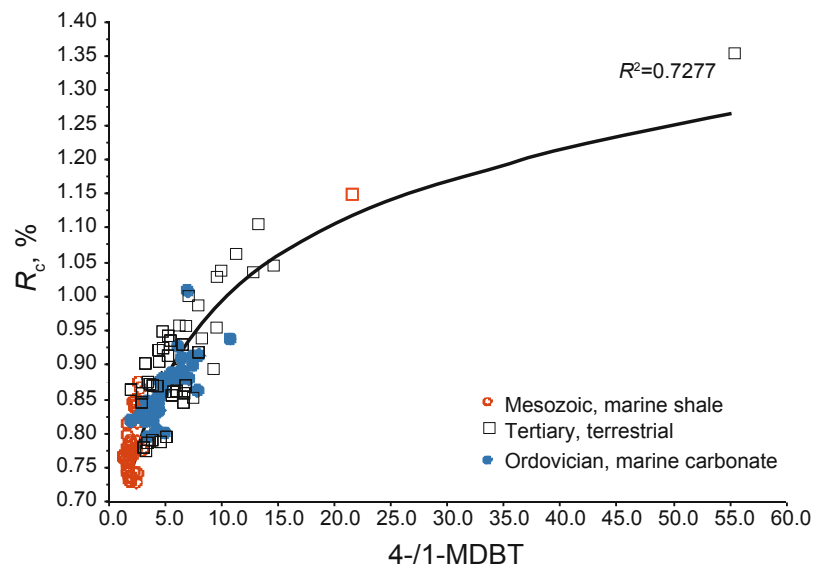

Fig. 4 Relationship of 4-/1-MDBT (MDR: 4-/1-methyldibenzothiophene) to vitrinite reflectance $R_{\mathrm{c}}(\%)$ calculated by MPI-1 (methylphenanthrene index) for oils in this study. $R_{\mathrm{c}}(\%)=0.4+0.6 \times \mathrm{MPI}-1$ for $\mathrm{MPR}<2.65$. MPR $=2$-/1-methylphenanthrene

The ratio of 4,6-/1,4-DMDBT shows a good correlation with MDR for each data set (Fig. 3(a) and 3(b)) and also exhibits an exponential relationship with $R_{\mathrm{c}}(\%)$ (Fig. 5). In this study, oils derived from marine carbonate and marine shale source rocks do not exhibit significant differences in the relationship between 4,6-/1,4-DMDBT and MDR or 2,4-/1,4-DMDBT (Fig. 3(a) and 3(b)). However, oils from terrestrial source rocks seem to have relatively lower 4,6-/1,4-DMDBT (Fig. 3(a) and 3(b), Fig. 5). Compared with oils from marine carbonate and shale source rocks, the oils from terrestrial Beibuwan Basin are systematically 0.5 lower (Fig. 5). This suggests that organic matter type and lithology of source rocks affect the 4,6-/1,4-DMDBT ratio even at advanced maturity stage. Thus, we cannot compare the maturity of oils originated from source rocks with different organic matter types and lithologies based solely on the 4,6-/1,4-DMDBT ratios. Calibration by other maturity parameters or investigation of DBT distribution in related source rocks seems to be necessary.

The DBT maturity parameters are based on the theoretical differences in thermodynamic stability of various methyl

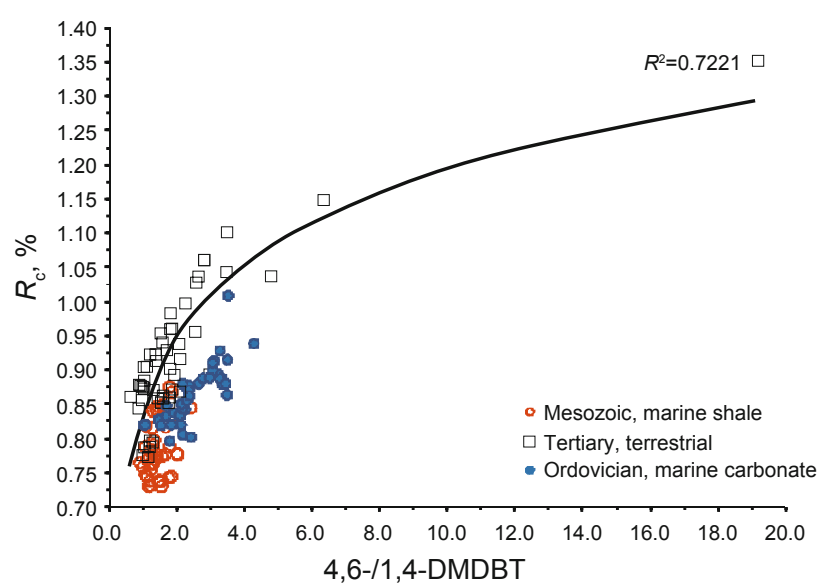

Fig. 5 Relationship of 4,6-/1,4-DMDBT (4,6-/1,4-dimethyldibenzothiophene) to vitrinite reflectance $R_{\mathrm{c}}(\%)$ calculated by MPI-1 (methylphenanthrene index) for oils in this study
DBT isomers. The enthalpy of formation $(\Delta \mathrm{H})$ is a commonly used thermodynamic parameter, and the lower the value, the greater the stability of that isomer (Budzinski et al, 1993). For example, the enthalpy of formation for 4-MDBT is 23,755 $\mathrm{cal} / \mathrm{mol}$, which is $5,979 \mathrm{cal} / \mathrm{mol}$ lower than that of 1 -MDBT, and the value for 4,6-DMDBT is $14,355 \mathrm{cal} / \mathrm{mol}$, which is also $5,979 \mathrm{cal} / \mathrm{mol}$ lower than that of 1,4-DMDBT (Richard, 2001). The enthalpy of formation for 2,4-DMDBT is 14,156 $\mathrm{cal} / \mathrm{mol}$, which is a little lower than that of 4,6-DMDBT. The isomers 2,6-, 3,6-DMDBT have the same $\Delta H$ values as that of 2,4-DMDBT (Richard, 2001), suggesting their similar thermodynamic stability. Thus, 2,6-/1,4- or 3,6-/1,4-DMDBT ratios are also potential maturity indicators similar to 2,4-/1,4-DMDBT. However, these two isomers cannot be completely separated in routine GC-MS analysis (Figs. 2 and $6)$. Thus, we propose a new maturity parameter $(2,6+3,6)$ / 1,4-DMDBT based on the relative abundance of DMDBT isomers.

Oil samples originated from typical terrestrial, marine carbonate and marine shale source rocks demonstrate that the newly proposed $(2,6+3,6)$ - / 1,4-DMDBT ratio correlates well with the MDR and 2,4-/1,4-DMDBT (Fig. 3(c) and $3(d)$ ) (with correlation coefficients in the range of 0.70 to 0.90 , and 0.87 to 0.94 , respectively), suggesting its similar behavior with increasing maturity. Like MDR, the $(2,6+$ $3,6)$-/1,4-DMDBT ratio for each data set also has similar trends with that of MDR or 2,4-/1,4-DMDBT (Fig. 3(c) and $3(\mathrm{~d}))$. In contrast with 4,6-/1,4-DMDBT, $(2,6+3,6)$ /1,4-DMDBT seems to have minor differences among oils with different origins, which can be clearly demonstrated in the relationship between $(2,6+3,6)-/ 1,4-\mathrm{DMDBT}$ and $R_{\mathrm{c}}(\%)$ (Fig. 7). Like 4,6-/1,4-DMDBT, $(2,6+3,6)-/ 1,4-D M D B T$ also has an exponential relationship with $R_{\mathrm{c}}(\%)$. However, unlike 4,6-/1,4-DMDBT, oils with different origins seem to exhibit a similar trend, suggesting that lithology and organic facies seem to have minor influences on the $(2,6+3,6)$ /1,4-DMDBT ratio for high mature oils. We can also infer that the organic facies and lithology may have different influences on different DBT based maturity parameters.

\subsection{Maturity parameters related to trimethyldibenzothiophene}

One maturity parameter TMDBT index based on trimethyldibenzothiophene (TMDBT) was previously proposed by Chakhmakhchev and Suzuki (1995). The ratio of two isomers in the $\mathrm{m} / \mathrm{z} 226$ mass chromatogram increases with maturity and has a good correlation with MDR, suggesting its similar behavior with increasing maturity. However, this empirical index has not been widely used for the unknown structures of these TMDBT isomers. They are simply named peak 3 and peak 5 (Fig. 1), respectively by Chakhmakhchev et al (1997). The progress on GC retention behavior of DBT on different stationary phase columns (Mössner et al, 1999; Schade and Andersson, 2006) provides a relatively reliable way to identify more isomers of trimethyldibenzothiophene. A series of TMDBT isomers were identified in oils, coal and sedimentary rock extracts ( $\mathrm{Li}$ et al, 2012) by comparing with retention indices reported in literature. In this study, some isomers were identified by comparing with the separation experiments of TMDBT isomers (Mössner et al, 1999), the 


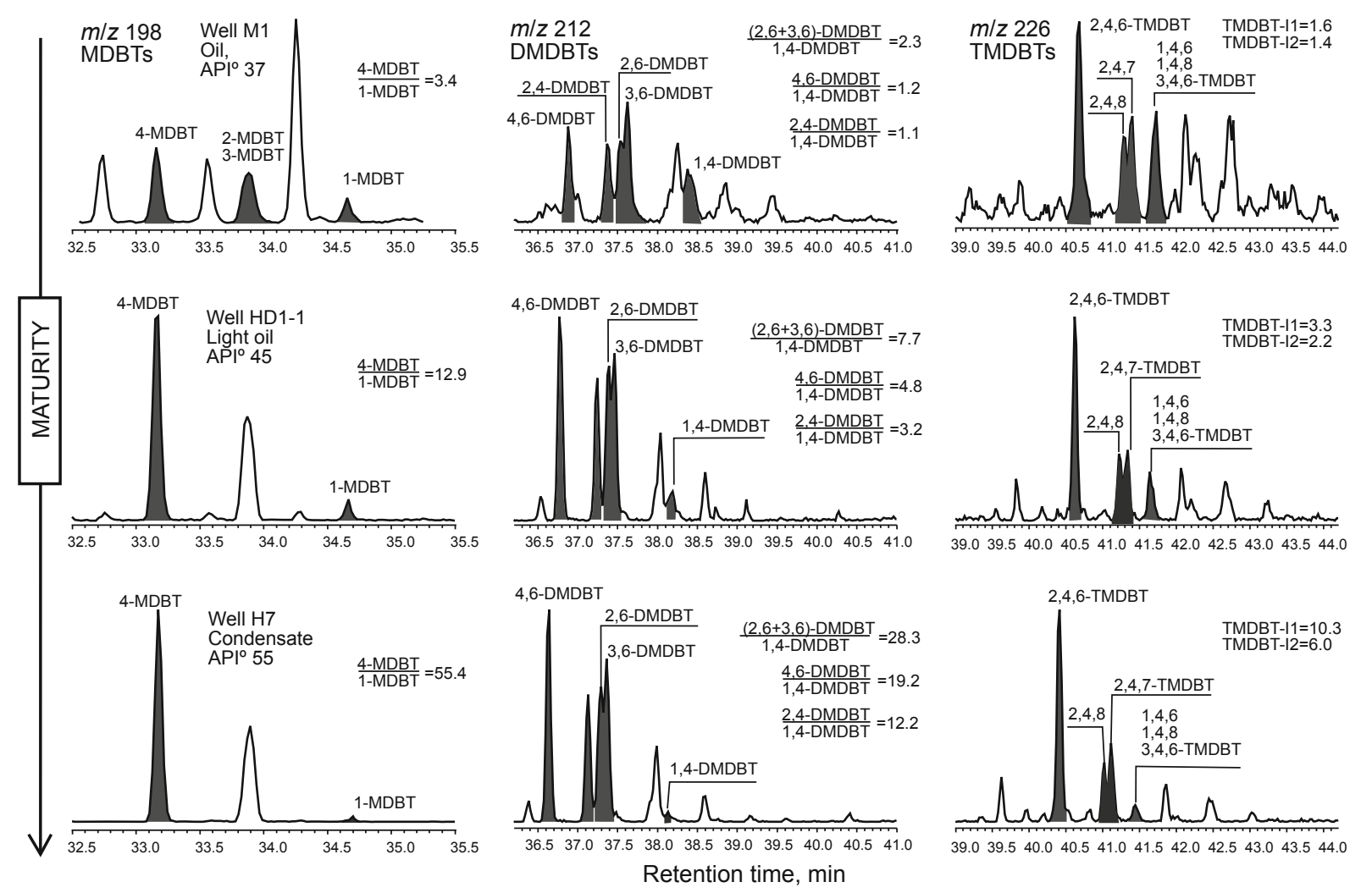

Fig. 6 Maturity effect on the distribution of methyl-, dimethyl-, and trimethyldibenzothiophenes (m/z 198, 212, and 226). Oil samples are from Beibuwan Basin, South China Sea. Note that 1,6-DMDBT isomer may co-elute with 1,4-DMDBT. Due to their similar thermodynamic stabilities, there is no significant influence on the application of 2,4-/1,4-DMDBT and 4,6-/1,4-DMDBT as maturity indicators

retention indices (Schade and Andersson, 2006) and co-elution of some internal standards. For example, the peak 3 in the $\mathrm{m} / \mathrm{z}$ 226 mass chromatogram by Chakhmakhchev et al (1997) is assigned as 2,4,6-TMDBT, and peak 5, as 1,4,6-, 1,4,8- and 3,4,6-TMDBT (Fig. 1). Richard (2001) did not provide the thermodynamic parameters for TMDBT isomers in his study. However, the thermodynamic stability for some isomers can be reasonably inferred. Budzinski et al (1991) reported that the 1 -position is less stable than the 3-position which is less

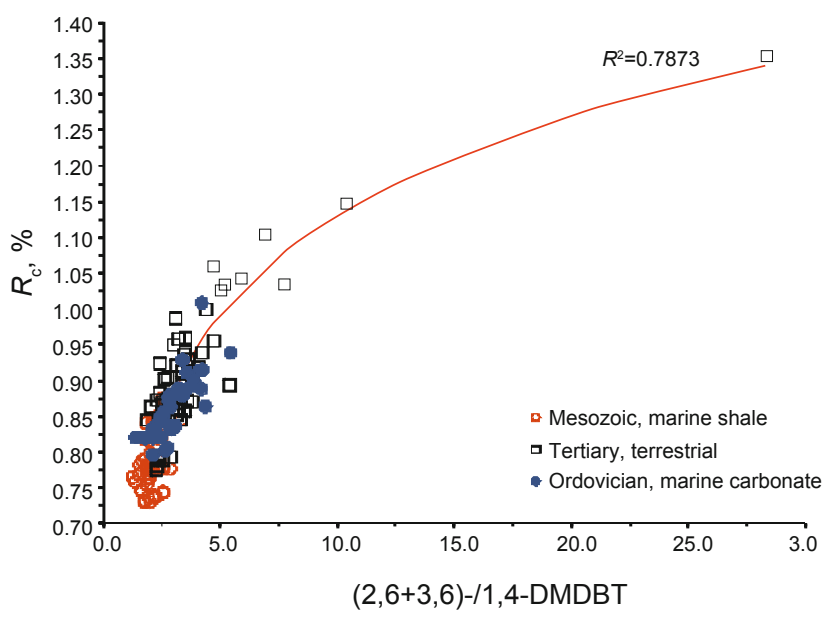

Fig. 7 Relationship of $(2,6+3,6)-/ 1,4$-DMDBT $((2,6+3,6)-/ 1,4-$ dimethyldibenzothiophene) to vitrinite reflectance $R_{\mathrm{c}}(\%)$ calculated by MPI1 (methylphenanthrene index) for oils in this study stable than the 2-position, and the positions 4- and 6- are more stable. Thus the 4,6-, 2,6- and 3,6-DMDBT isomers are more stable and are the main isomers in mid-mature to high-mature oils. According to this rule, the 2,4,6-TMDBT may be the most stable isomer among all TMDBT isomers. Our observations also suggest that peak 3, i.e. 2,4,6-TMDBT for all oils in this study is the most abundant isomer (Figs. 1 and 6). Therefore, the empirical TMDBT index proposed by Chakhmakhchev and Suzuki (1995) and Chakhmakhchev et al (1997) is reliable on a thermodynamic basis.

The TMDBT index (TMDBT-I1 in our study) here defined as 2,4,6-/(1,4,6+1,4,8+3,4,6)-TMDBT for 140 oil samples from three basins is listed in Tables 1 and 2. It also exhibits a good correlation with MDR and 2,4-/1,4-DMDBT ratio with coefficients in the range of 0.76 to 0.91 for MDR and 0.75 to 0.93 for 2,4-/1,4-DMDBT (Fig. 8(a) and 8(b)). Unlike 4,6-/1,4-DMDBT or $(2,6+3,6)$-/1,4-DMDBT, oils from terrestrial and marine shale have similar trends with MDR or 2,4-/1,4-DMDBT, and they are relatively lower than those of marine carbonate sourced oil. Thus, we cannot compare the maturity of oils with different origin by TMDBT-I1 without any calibration. The distributions of methylated PAHs in oils and sedimentary organic matter are controlled by complicated chemical processes, such as methylation, demethylation, and disproportionation reactions (methyl shifting) (Alexander et al, 1985; Strachan et al, 1988; van Aarssen et al, 1999; Bastow et al, 2000). Thus, further study is needed to investigate the formation and transformation of polycyclic aromatic sulfur hydrocarbons with the increasing thermal 

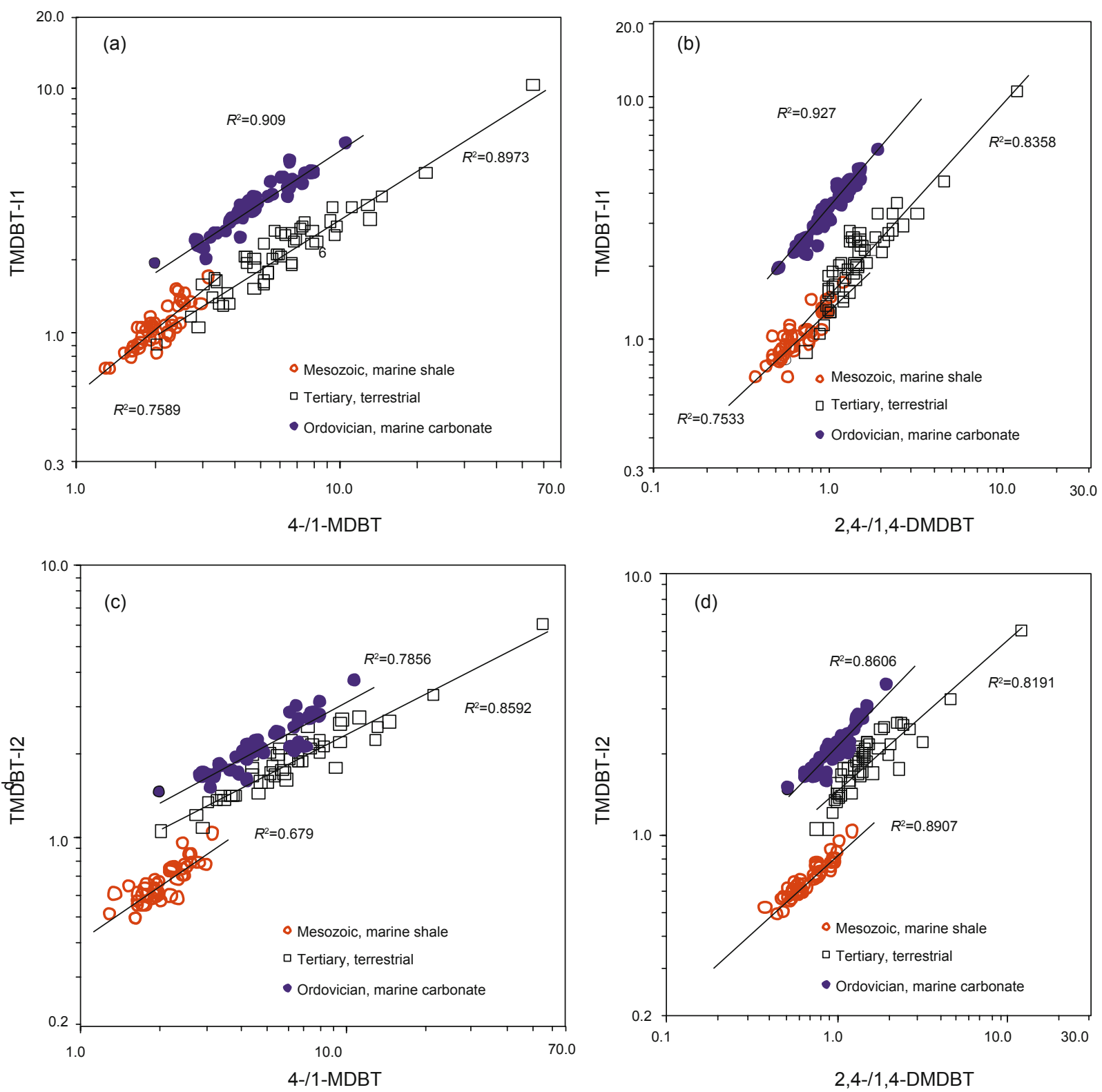

Fig. 8 Cross plots showing a good correlation between (a) TMDBT-I1 and 4-/1-MDBT ratio, (b) TMDBT-I1 and 2,4-/1,4-DMDBT ratio, (c) TMDBT-I2 and 4-/1-MDBT ratio, and (d) TMDBT-I2 and 2,4-/1,4-DMDBT ratio

stress, especially during diagenesis.

Other TMDBT related ratios applicable for maturity assessments can be proposed on the basis of TMDBT thermal stability. This study proposes a new TMDBT maturity parameter TMDBT-2, which is defined as $(2,4,8+2,4,7)-/$ $(1,4,6+1,4,8+3,4,6)$-TMDBT ratio (Tables 1 and 2, Fig. 6). Data of 140 oil samples in our study demonstrate that it correlates well with other DBT maturity parameters, such as MDR or 2,4-/1,4-DMDBT (Fig. 8(c) and 8(d)) with correlation coefficients in the range of 0.68 to $0.86,0.82$ to 0.89 , respectively. Our study together with previous studies (e.g. Chakhmakhchev et al, 1997) shows that all thermodynamic stability based DBT maturity parameters correlate well with each other, suggesting their similar behavior with increasing maturity. Like TMDBT-I1 and 4,6-/1,4-DMDBT ratio, TMDBT-I2 is also related to the organic facies or/and depositional environment (Fig. 8(c) and $8(d)$ ). Thus, the same TMDBT-I2 values for oils from different basins may not indicate similar thermal maturity.

The maturity parameters based on the distributions of DBT work well over a relatively wide range of maturity. They are effective maturity indicators for oils with moderately mature to high mature petroleum, especially at an advanced stage of thermal transformation (Chakhmakhchev et al, 1997). They are usually present in significant concentrations and can be easily identified and measured in light oils and condensates depleted in biomarkers. However, with increasing maturity, the thermodynamically unstable isomers such as 1-MDBT may be absent or present in extremely low concentrations below the detection limit in GC-MS analyses. In our study, the 1-MDBT and 1,4-DMDBT isomers for condensate from Well $\mathrm{H} 7$ in the Beibuwan Basin are present in extremely low concentration (Fig. 6), which may result in inaccurate values of the MDR and other 1,4-DMDBT related maturity 
parameters.

The MPI-1 of this condensate is as high as 1.59 and the MPR (2-/1-methylphenanthrene) gets to 3.77 (Fig. 9). According to Radke and Welte (1981) and Radke et al (1986), when the $R_{\mathrm{c}}$ is higher than $1.35 \%$ or MPR $>2.65$, the MPI- 1 shows a reverse trend with the increasing maturity and the $R_{\mathrm{c}}$ (\%) could be calculated using another empirical formula $R_{\mathrm{c}}$ $(\%)=2.3-0.6 \times$ MPI- 1 . Here we found that the $R_{\mathrm{c}}(\%)$ values calculated by both equations are nearly the same $(1.35 \%)$. It may just correspond to the inflection point for MPI against $R_{\mathrm{c}} \%$ relationship (Fig. 9). This coincides with the upper level of maturity that can be evaluated by DBT $\left(R_{\mathrm{o}} \approx 1.35 \%-1.5 \%\right.$ by Chakhmakhchev et al, 1997 , and $R_{\mathrm{o}}=1.35 \%$ by Radke and Welte, 1981).

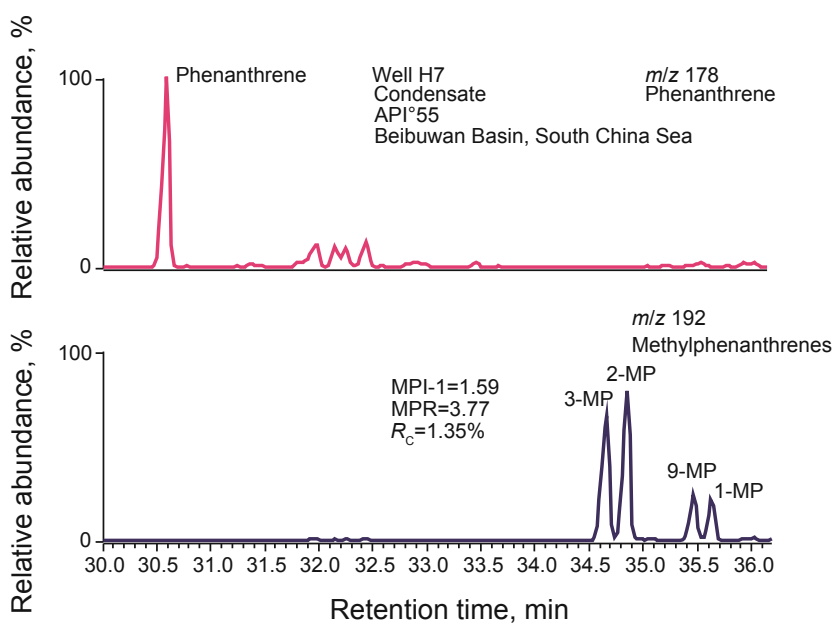

Fig. 9 The m/z 178 and 192 mass chromatograms for the condensate from Well H7 in the Beibuwan Basin, South China Sea show the distribution of phenanthrene and methylphenanthrenes.

Note: 3-MP: 3-methylphenanthrene; MPI-1 $=1.5 \times$ (Phenanthrene + $2-\mathrm{MP}+3-\mathrm{MP}) /\left(\right.$ Phenanthrene + 9-MP + 1-MP); MPR $=2-\mathrm{MP} / 1-\mathrm{MP} ; R_{\mathrm{c}}$ $(\%)=2.3-0.6 \times$ MPI-1 (Radke and Welte, 1981)

The TMDBT-based maturity parameter TMDBT-I2 proposed in this study and previous TMDBT-I1 appear to be more reliable for over-mature light oils and condensates. The thermodynamically unstable 1,4,6-, 1,4,8- and 3,4,6-TMDBT isomers have relatively higher concentration (Fig. 2) at high maturity.

Up to now, there is little knowledge about the formation of DBTs during diagenesis. Like most of the PAHs in oil and ancient sedimentary organic matter, DBTs do not have certain biological precursors. They were mainly produced by reaction with other compounds, e.g. biphenyl and sulfur (Asif et al, 2009). Understanding the formation and evolution processes of DBTs during diagenesis will be very important to the application of maturity parameters based on DBT in low mature oils.

\section{Conclusions}

The structures of a series of trimethyldibenzothiophenes (TMDBTs) have been firmly assigned by comparison with reported retention indices and GC-MS separation experiments in this study. A previously proposed TMDBT index that is based on the relative abundances of two TMDBT isomers (peak 3 to peak 5) with unknown structures is here identified as four isomers and defined as 2,4,6-/(1,4,6+1,4,8+ $3,4,6)$-TMDBT. Another maturity parameter $(2,4,7+2,4,8)-/$ $(1,4,6+1,4,8+3,4,6)$-TMDBT based on TMDBT is proposed in light of the same thermodynamic principles. Both these indices (TMDBT-I1 and TMDBT-I2) correlate well with the MDR and 2,4-/1,4-DMDBT, displaying their high potential for maturity evaluation, especially at elevated maturity stages.

A new maturity parameter $(2,6+3,6)-/ 1,4-D M D B T$ relative to dimethyldibenzothiophene is also proposed based on their same thermodynamic stability as that of 2,4-DMDBT isomers. This ratio exhibits a good correlation with MDR and 2,4-/1,4-DMDBT, suggesting its similar behavior with increasing maturity. Unlike 4,6-/1,4-DMDBT ratio, oils with different origins seem to exhibit a similar trend, suggesting that lithology and organic facies seem to have minor influence on the $(2,6+3,6)-/ 1,4-D M D B T$ ratio for high mature oils.

DBTs distribution indicators would be widely applied in maturity assessments in oil and source rocks since they appear to work well over a relatively high range of maturity, especially for over-mature light oils and condensates lacking biomarkers.

\section{Acknowledgements}

The work was funded by the National Natural Science Foundation of China (Grant No. 41272158) and the State Key Laboratory of Petroleum Resources and Prospecting (PRP/indep-2-1302). The authors thank the assistance of Zhu Dan and Shi Quan in the GC-MS analyses. This paper benefits greatly from the discussion with Drs. Paul G. Lillis and Geoffrey S. Ellis at U.S. Geological Survey. Dr. Paul G. Lillis is greatly appreciated for his assistance in polishing the manuscript. The author Li Meijun is highly indebted to Dr. Geoffrey S. Ellis for his assistance during Dr Li's stay as a visiting scholar at the U.S. Geological Survey. The authors would like to thank two anonymous reviewers for their comments and constructive suggestions which significantly improved the quality of this manuscript.

\section{References}

Alexander R, Kagi R I, Rowland S J, et al. The effects of thermal maturity on distributions of dimethylnaphthalenes and trimethylnaphthalenes in some ancient sediments and petroleums. Geochimica et Cosmochimica Acta. 1985. 49(2): 385-395

Asif M, Alexander R, Fazelat T, et al. Geosynthesis of dibenzothiophene and alkyldibenzothiophenes in crude oils and sediments by carbon catalysis. Organic Geochemistry. 2009. 40: 895-901

Asif M. Geochemical applications of polycyclic aromatic hydrocarbons in crude oils and sediments from Pakistan. Ph.D. thesis, University of Engineering and Technology, Lahore, Pakistan. 2010

Bao J P, Wang T.-G and Chen F J. Relative abundance of alkyl dibenzothiophenes in the source rocks and their geochemical significances. Journal of China University of Petroleum. 1996. 20: 19-23 (in Chinese)

Bao J P and Zhu C S. The effects of biodegradation on the compositions of aromatic hydrocarbons and maturity indicators in biodegraded oils from Liaohe Basin. Science in China Series D: Earth Sciences. 2009. 52: 59-68 
Bastow T P, Alexander R, Fisher S J, et al. Geosynthesis of organic compounds. Part V - methylation of alkylnaphthalenes. Organic Geochemistry. 2000. 31(6): 523-534

Budzinski H, Garrigues P, Connan J, et al. Determination of maturity indicators in alkylated aromatic series by gas chromatographymass spectrometry (GC-MS). In: Manning D.A.C. (Ed.), Organic Geochemistry: Advances and Applications in Energy and the Natural Environment. Manchester University Press. 1991. 619-623

Budzinski H, Garrigues P, Radke M, et al. Use of molecular modeling as a tool to evaluate thermodynamic stability of alkylated polycyclic aromatic hydrocarbons. Energy \& Fuels. 1993. 7(4): 505-511

Chakhmakhchev A and Suzuki N. Saturate biomarkers and aromatic sulfur compounds in oils and condensates from different source rock lithologies of Kazakhstan, Japan and Russia. Organic Geochemistry. 1995. 23: 289-299

Chakhmakhchev A, Suzuki M and Takayama K. Distribution of alkylated dibenzothiophenes in petroleum as a tool for maturity assessments. Organic Geochemistry. 1997. 26: 483-490

Connan J, Brououllec J, Dessort D, et al. The microbial input in carbonate-anhydrite facies of Sabkha palaeoenvironment from Guatemala: A molecular approach. In: Leythhaeuser D., Rullkötter J. (Eds.), Advances in Organic Geochemistry. Organic Geochemistry. 1986. 10: $29-50$

Cui J, Wang T, Li M, et al. Oil filling history of the Bashituo Oilfield in the Markit Slope, SW Tarim Basin, China. Petroleum Science. 2013. 10(1): 58-64

Ding W X, Wang W and Ma Y. Characteristics of Liushagang Formation petroleum system in Fushan Depression of Beibuwan Basin. Offshore Petroleum. 2003. 23(2): 1-6 (in Chinese)

Douglas A G and Mair B J. Sulfur: role in genesis of petroleum. Science. 1965. 147: 99-501

Dzou L P, Noble R A and Senftle J T. Maturation effects on absolute biomarker concentration in a suite of coals and associated vitrinite concentrates. Organic Geochemistry. 1995. 10: 29-50

Farrington J W, Davis A C, Tarafa M E, et al. Bitumen molecular maturity parameters in the Ikpikpuk well, Alaskan North Slope. Organic Geochemistry. 1988. 13(1-3): 303-310

Gransch J A and Posthuma J. On the origin of sulphur in crudes. Advances in Organic Geochemistry. 1974. 727-739

Hanson W E. Origin of petroleum. In: Gruse W.A., Stevens D.R. (Eds.), Chemical Technology of Petroleum. New York: McGraw-Hill. 1960. Chap. 5, p. 247

Ho T Y, Rogers M A, Drushel H V, et al. Evolution of sulfur compounds in crude oils. AAPG Bulletin. 1974. 58(11): 2338-2348

Huang $\mathrm{H}$ and Pearson M J. Source rock palaeoenvironments and controls on the distribution of dibenzothiophenes in lacustrine crude oils, Bohai Bay Basin, eastern China. Organic Geochemistry. 1999. 30: $1455-1470$

Hughes W B. Use of thiophenic organosulfur compounds in characterizing crude oils derived from carbonate versus siliclastic sources. In: Palacas J.B. (Ed.), Petroleum Geochemistry and Source Rocks Potential of Carbonate Rocks. AAPG Studies in Geology No.18, Oklahoma, AAPG Press. 1984. 181-196

Hughes W B, Holba A G and Dzou L I P. The ratios of dibenzothiophene to phenanthrene and pristane to phytane as indicators of depositional environment and lithology of petroleum source rocks. Geochemica et Cosmochimica Acta. 1995. 59: 3581-3598

Kruge M A. Determination of thermal maturity and organic matter type by principal components and analysis of the distributions of polycyclic aromatic compounds. International Journal of Coal Geology. 2000. 43: 27-51

Lee M L, Vassilaros D L, White C M, et al. Retention indices for programmed-temperature capillary-column gas chromatography of polycyclic aromatic hydrocarbons. Journal of Chromatography A.
1979. 51(6): 768-774

Li M J, Wang T G, Liu J, et al. Oil charging orientation and accumulation characteristics of oil reservoirs in the Fushan Sag, Beibuwan Basin. Petroleum Geology and Experiment. 2007a. 29(2): 172-176 (in Chinese)

Li M, Simoneit B R T, Zhong N, et al. The distribution and origin of dimethyldibenzothiophenes in sediment extracts from the Liaohe Basin, East China. Organic Geochemistry. 2013b. 65: 63-73

Li M, Wang T, Liu J, et al. Characteristics of oil and gas accumulation in Yong'an-Meitai Area of the Fushan Depression, Beibuwan Basin, South China Sea. Petroleum Science. 2007b. 4(4): 23-33

Li M, Wang T, Liu J, et al. Total alkyl dibenzothiophenes content tracing the filling pathway of condensate reservoir in the Fushan Depression, South China Sea. Science in China Series D: Earth Sciences. 2008a. 51(Supp.II): 138-145

Li M, Wang T, Liu J, et al. Occurrence and origin of carbon dioxide in the Fushan Depression, Beibuwan Basin, South China Sea. Marine and Petroleum Geology. 2008b. 25(6): 500-513

Li M, Wang T, Liu J, et al. The occurrence of oleananes in the Beibuwan Basin and its application to the study of maturity and oil-source rock correlation. Acta Geologica Sinica (English Edition). 2008c. 82(3): 585-595.

Li M, Wang T, Liu J, et al. Alkyl naphthalenes and phenanthrenes: molecular markers for tracing filling pathways of light oil and condensate reservoirs. Acta Geologica Sinica (English Edition). 2010. 84(5): 1294-1305

Li M, Wang T, Simoneit B R T, et al. Qualitative and quantitative analysis of dibenzothiophene, its methylated homologues, and benzonaphthothiophenes in crude oils, coal, and sediment extracts. Journal of Chromatography A. 2012. 1233: 126-136

Li M, Wang T, Zhong N, et al. Ternary diagram of fluorenes, dibenzothiophenes and dibenzofurans: Indicating depositional environment of crude oil source rocks. Energy Exploration \& Exploitation. 2013c. 31: 569-588

Li M, Zhong N, Shi S, et al. The origin of trimethyldibenzothiophenes and their application as maturity indicators in sediments from the Liaohe Basin, East China. Fuel. 2013a. 103: 299-307

Li S, Pang X, Shi Q, et al. Origin of the unusually high dibenzothiophene concentrations in Lower Ordovician oils from the Tazhong Uplift, Tarim Basin, China. Petroleum Science. 2011. 8(4): 382-391

Luo J, Cheng K M, Fu L X, et al. Alkylated dibenzothiophene Index-a new method to assess thermal maturity of source rocks. Acta Petrolei Sinica. 2001. 22(3): 27-31 (in Chinese)

Moldowan J M, Dahl J, Huizinga B J, et al. The molecular fossil record of oleanane and its relation to angiosperms. Science. 1994. 265: 768-771

Mössner S G, de Alda M J L, Sandera L C, et al. Gaschromatographic retention behavior of polycyclicaromatic sulfur heterocyclic compounds, (dibenzothiophene, naphtho[b]thiophenes, benzo[b] naphthothiophenes and alkyl-substituted derivatives) on stationary phases of different selectivity. Journal of Chromatography A. 1999. 841(2): 207-228

Murray A P, Sosrowidjojo I B, Alexander R, et al. Oleananes in oils and sediments: Evidence of marine influence during early diagenesis. Geochemica et Cosmochimica Acta. 1997. 61(6): 1261-1276

Orr W L. Kerogen/asphaltene/sulfur relationships in sulfur-rich Monterey oils. Organic Geochemistry. 1986. 10: 499-516

Payzant J D, Mojelsky T W and Strausz O P. Improved methods for the selective isolation of the sulfide and thiophenic classes of compounds from petroleum. Energy \& Fuels. 1989. 3(4): 449-454

Peters K E, Walters C C and Moldowan J M. The Biomarker Guide $\left(2^{\text {nd }}\right.$ ed). New York: Cambridge University Press. 2005

Radke M. Application of aromatic compounds as maturity indicators in source rocks and crude oils. Marine and Petroleum Geology. 1988. 5: 
224-236

Radke $\mathrm{M}$ and Welte D H. The methylphenanthrene index (MPI): a maturity parameter based on aromatic hydrocarbons. In: Bjoroy M. (Eds.), Advances in Organic Geochemistry. 1981. Chichester: Wiley. 504-512

Radke $M$ and Willsch H. Extractable alkyldibenzothiophenes in Posidonia Shale (Toarcian) source rocks: Relationship of yields to petroleum formation and expulsion. Geochimica et Cosmochimica Acta. 1994. 58(23): 5223-5244

Radke M, Horsfield B, Littke R, et al. Maturation and petroleum generation. In: Welte D.H., Horsfield B., Baker D.R. (Eds.), Petroleum and Basin. Verlag: Springer. 1997. 171-229

Radke M, Vriend S P and Ramanampisoa L R. Alkydibenzofurans in terrestrial rocks: Influence of organic facies and maturation. Geochimica et Cosmochimica Acta. 2000. 64(2): 275-286

Radke M, Welte D H and Willsch H. Maturity parameters based on aromatic hydrocarbons: Influence of the organic matter type. Organic Geochemistry. 1986. 10(1-3): 51-63

Radke M, Welte D H and Willsch H. Distribution of alkylated aromatic hydrocarbons and dibenzothiophenes in rocks of the Upper Rhine Graben. Chemical Geology. 1991. 93: 325-341

Radke M, Willsch H, Leythaeuser, et al. Aromatic components of coal: relation of distribution pattern to rank. Geochimica et Cosmochimica Acta. 1982. 46(10): 1831-1848

Richard L. Calculation of the standard molal thermodynamic properties as a function of temperature and pressure of some geochemically important organic sulfur compounds. Geochimica et Cosmochimica Acta. 2001. 65: 3827-3877

Rowland S J, Alexander R, Kagi R I, et al. Microbial degradation of aromatic components of crude oils: A comparison of laboratory and field observations. Organic Geochemistry. 1986. 9(4): 153-161

Santamaría-Orozco D, Horsfield B, Primio R D I, et al. Influence of maturity on distributions of benzo- and dibenzothiophenes in Tithonian source rocks and crude oils, Sonda de Campeche, Mexico. Organic Geochemistry. 1998. 28: 423-439
Schade T and Andersson J T. Speciation of alkylated dibenzothiophenes through correlation of structure and gas chromatographic retention indexes. Journal of Chromatography A. 2006. 1117: 206-213

Schou L and Myhr M B. Sulfur aromatic compounds as maturity parameters. Organic Geochemistry. 1988. 13(1-3): 61-66

Shen P, Wang W, Zhang X, et al. Differentiating depositional environments and maturity of crude oils using GC-AED benzothiophenic spectra. Science in China Series D: Earth Sciences. 1998. 41(4): 401-407

Strachan M G, Alexander R and Kagi R I. Trimethylnaphthalenes in crude oils and sediments: Effects of source and maturity. Geochimica et Cosmochimica Acta. 1988. 52(5): 1255-1264

van Aarssen B G K, Bastow T P, Alexander R, et al. Distributions of methylated naphthalenes in crude oils: indicators of maturity, biodegradation and mixing. Organic Geochemistry. 1999. 30(10): 1213-1227

Vassilaros D L, Kong R C, Later D W, et al. Linear retention index system for polycyclic aromatic compounds: Critical evaluation and additional indices. Journal of Chromatography A. 1982. 252: 1-20

Wan L, Liu J, Mao F, et al. The petroleum geochemistry of the Termit Basin, Eastern Niger. Marine and Petroleum Geology. 2014. 51: 167-183

Wang T.-G, He F, Li M, et al. Alkyl-dibenzothiophenes: molecular tracers for filling pathway in oil reservoirs. Chinese Science Bulletin. 2004. 49: 2399-2404

Wang T.-G, He F, Wang C, et al. Oil filling history of the Ordovician oil reservoir in the major part of the Tahe Oilfield, Tarim Basin, NW China. Organic Geochemistry. 2008. 38: 1637-1646

Xia Y and Zhang G. Investigation of mechanisms of formation of biphenyls and benzonaphthothiophenes by simulation experiment. Science in China Series D: Earth Science. 2002. 45(5): 392-398

Zhang S and Huang H. Geochemistry of Palaeozoic marine petroleum from the Tarim Basin, NW China: Part 1. Oil family classification. Organic Geochemistry. 2005. 36: 1204-1214

(Edited by Hao Jie) 